\title{
Cosmic Strings and Their Induced Non-Gaussianities in the Cosmic Microwave Background
}

\author{
Christophe Ringeval \\ Institute of Mathematics and Physics, Centre for Cosmology, Particle Physics and Phenomenology, Louvain University, \\ 2 Chemin du Cyclotron, 348 Louvain-la-Neuve, Belgium
}

Correspondence should be addressed to Christophe Ringeval, ringeval@fyma.ucl.ac.be

Received 15 January 2010; Accepted 27 May 2010

Academic Editor: Eiichiro Komatsu

Copyright ( $) 2010$ Christophe Ringeval. This is an open access article distributed under the Creative Commons Attribution License, which permits unrestricted use, distribution, and reproduction in any medium, provided the original work is properly cited.

\begin{abstract}
Motivated by the fact that cosmological perturbations of inflationary quantum origin were born Gaussian, the search for nonGaussianities in the cosmic microwave background (CMB) anisotropies is considered as the privileged probe of nonlinear physics in the early universe. Cosmic strings are active sources of gravitational perturbations and incessantly produce non-Gaussian distortions in the CMB. Even if, on the currently observed angular scales, they can only contribute a small fraction of the $\mathrm{CMB}$ angular power spectrum, cosmic strings could actually be the main source of its non-Gaussianities. In this paper, after having reviewed the basic cosmological properties of a string network, we present the signatures Nambu-Goto cosmic strings would induce in various observables ranging from the one-point function of the temperature anisotropies to the bispectrum and trispectrum. It is shown that string imprints are significantly different than those expected from the primordial type of nonGaussianity and could therefore be easily distinguished.
\end{abstract}

\section{Motivations}

The origin of cosmic strings dates back to the discovery that cosmological phase transitions triggered by the spontaneous breakdown of the fundamental interaction symmetries may form topological defects [1-3]. Cosmic strings belong to the class of line-like topological defects, as opposed to pointlike monopoles and the membrane shaped domain walls. As shown by Kibble, the appearance of defects in any field theory is related to the topology of the vacuum manifold [3]. If the ground state of a field theory experiences a spontaneous breakdown from a symmetry group $G$ to a subgroup $H$, Kibble showed that cosmic strings will be formed if the first homotopy group $\pi_{1}(G / H) \neq I$ is nontrivial. In other words, if noncontractile loops can be found in the manifold $\mathcal{M}=$ $G / H$ of equivalent vacua. Similarly, the other homotopy groups $\pi_{0}$ and $\pi_{2}$ determine the formation of domain walls and monopoles, respectively. Once formed and cooled, these defects cannot be unfolded, precisely due to their nontrivial topological configuration over the vacuum manifold of the theory. This simple statement suggests that cosmic strings, and topological defects in general, are a natural outcome of the unification of the fundamental interactions in the context of Cosmology. As remnants of unified forces, their discovery would be an incredible opportunity to probe extremely highenergy physics with "a telescope".

In the last thirty years, many works have been devoted to the cosmological consequences, signatures, and searches for topological defects [4-7]. They have pushed cosmic strings to the privileged place to be generically compatible with observations. Indeed, domain walls and monopoles are prone to suffer from the cosmological catastrophe problem; their formation is sufficiently efficient (or their annihilation sufficiently inefficient) to either overclose the universe or spoil the Big-Bang Nucleosynthesis (BBN) predictions $[8,9]$. For domain walls, this implies that either they should be extremely light, that is, formed at an energy scale less than a few $\mathrm{MeV}$, or no discrete symmetry should have been broken during the cooling of the universe. There is not so much choice for the monopoles; if interactions were unified, monopoles would have been formed. The homotopy group of $\pi_{2}(G / H)$ with $H$ containing the $U(1)$ of electroweak interactions is indeed nontrivial.(As often with topological defects, sensitivity to the underlying model is such that one 
can often find a counter-example of any result. Both of these statements, on walls and monopoles, can be evaded in some particular models or with some amount of finetuning, as for instance if cosmic strings can be attached to them and catalyse annihilations $[10,11]$.) Cosmic inflation was originally designed to solve the monopole problem. If a phase of accelerated expansion of the universe occurs, then any defects will be diluted enough to no longer have any (dramatic) consequences on cosmology [12-15]. Meanwhile, Inflationary Cosmology solves the flatness and homogeneity problem of the standard Big-Bang model, explains the origin and spectrum of the cosmic microwave background (CMB) anisotropies, as the formation of the large-scale structures [16-19]. Inflation provides a priori an easy solution to the topological defects problem by diluting them to at most one per Hubble radius. However, one has to keep in mind that this mechanism works only if the defects were formed before inflation, and even in that case some may survive [20]. This has to be the case for monopoles and heavy walls, but not for local strings. On the contrary, exhaustive analysis of particle physics motivated inflationary models, embedding the Standard Model $S U(3) \times S U(2) \times$ $U(1)$, has shown that strings are generically produced at the end of inflation [21]. In this picture, our universe should contain cosmic strings whose properties are closely related to those of the inflation [22-24]. String Theory provides an alternative framework to Field Theories: brane inflationary models propose that the accelerated expansion of the universe is induced by the motion of branes in warped and compact extradimensions [25-28]. Inflation ends when two branes collide and such a mechanism again triggers the formation of one-dimensional cosmological extended objects, dubbed cosmic superstrings [29-32]. These objects may be cosmologically stretched fundamental strings or onedimensional D-brane $[33,34]$. Although cosmic superstrings are of a different nature than their topological analogue, they produce the same gravitational effects and share similar cosmological signatures $[35,36]$.

Among the expected signatures, cosmic strings induce temperature anisotropies in the $\mathrm{CMB}$ with an amplitude typically given by $G U$, where $U$ is the string energy per unit length and $G$ the Newton constant [37].(To avoid any confusion with Greek tensor indices, we will use the Carter's notations $U$ and $T$ for the string energy density and tension [38].) For the Grand Unified Theory (GUT) energy scale, one has $G U \simeq 10^{-5}$, which precisely corresponds to the observed amplitude of the CMB temperature fluctuations [39]. However, the power spectra do not match; topological defects are active sources of gravitational perturbations, that is, they produce perturbations all along the universe history, and cannot produce the characteristic coherent patterns of the acoustic peaks [40-44]. Current CMB data analyses including a string contribution suggest that they can only contribute to at most $10 \%$ of the overall anisotropies on the observed angular scales $[45,46]$. For Abelian cosmic strings (see Section 2), numerical simulations in FriedmannLemaître-Robertson-Walker (FLRW) spacetimes show that this corresponds to an upper two-sigma bound $G U<7 \times$ $10^{-7}$ [47]. Direct detection searches provide less stringent limits but are applicable to all cosmic string models: $G U<$ $4 \times 10^{-6}[48-50]$. Detecting cosmic strings in the $\mathrm{CMB}$ certainly requires one to go further than the power spectrum $[51,52]$ (see, however, Section 4.5). In fact, strings induce line-like discontinuities in the CMB temperature through the so-called Gott-Kaiser-Stebbins effect, which are intrinsically of non-Gaussian nature $[53,54]$. In the inflationary picture, cosmological perturbations find their origin in the quantum fluctuations of the field-metric system, and therefore were born generically Gaussian. Non-Gaussianities can nevertheless appear from non-linear effects during inflation or from couplings to other fields (see the other articles in this issue). These non-Gaussianities are of the primordial type, that is, they exist before the cosmological perturbations reenter the Hubble radius. On the other hand, cosmic strings are a source of non-Gaussianity at all times and, as we will see, produce different signals from the $\mathrm{CMB}$ point of view. (Notice that second-order perturbations, being nonlinear, actively generate non-Gaussianities but at a relatively small amplitude [55-58].)

In this paper, we review the non-Gaussian features a cosmological network of cosmic strings produce in the $\mathrm{CMB}$ anisotropies. In a first section, we briefly scan various cosmic string models and emphasize their similarities and differences for cosmology. Making observable predictions for cosmic strings faces the problem of understanding their cosmological evolution. Not only one has to solve the local dynamics in curved space, but as extended objects, cosmic strings follow a globally nonlocal evolution: the fate of one string depends on its interactions with the others. The cosmological evolution of a network of cosmic strings is a nontrivial problem which can be overcome by means of numerical simulations. These simulations permit an estimation of the various statistical properties affecting the observational signatures, such as the number of strings per Hubble radius, their shapes, velocities, or the loop density distribution. Latest results in this area, for the Nambu-Goto (NG) type of cosmic strings, are presented in Section 3. Once the statistical properties of a cosmological cosmic strings network are known, it is possible to extract meaningful observables depending only on the unique model parameter $U$. (If no currents are flowing along the string, Lorentz invariance implies that the string tension $T$ equals the energy density U.) In Section 4, we recap the expected CMB temperature anisotropies induced by cosmic strings, derived from various methods. Particular attention is paid to small angle CMB maps which preserve all of the projected statistical information. We then derive the cosmic string signals expected in various non-Gaussian estimators ranging from the one-point function of the $\mathrm{CMB}$ temperature fluctuations to the bispectrum and trispectrum. We conclude in Section 5 and discuss various non-Gaussian aspects which still have to be explored.

\section{Cosmic Strings of Various Origins}

Cosmic strings of cosmological interest can be of various kinds depending on the microscopic model they stem from. 


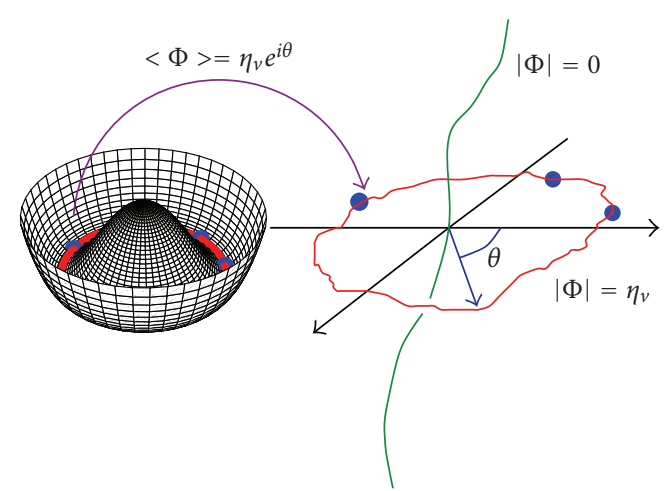

Figure 1: The Abelian Higgs potential in the complex plane $[\Re(\Phi), \Im(\Phi)]$. The nontrivial phase mapping from the internal space to the physical space (right) leads to the formation of a cosmic string. The old vacuum $|\Phi|=0$ becomes trapped inside the new one $|\Phi|=\eta_{\mathrm{v}}$.

As mentioned in the introduction, they can either be nontrivial stable, or metastable, field configurations or more fundamental objects in String Theory. From a gravitational point of view, they all are, however, line-like energy density and pressure distributions. In the following, we briefly review the different kinds of string having a cosmological interest and we emphasize their similarities and differences.

2.1. Abelian Vortices. The simplest example of cosmic string illustrating the Kibble mechanism is the Abelian Higgs model. The theory is invariant under a local gauge group $U(1)$ and the Higgs potential assumes its standard Mexican hat renormalisable form

$$
V(\Phi)=\frac{\lambda}{8}\left(|\Phi|^{2}-\eta_{\mathrm{v}}^{2}\right)^{2}
$$

where $\lambda$ is the self-coupling constant and $\eta_{\mathrm{v}}$ the vacuum expectation value of the Higgs field $\Phi$. In Minkowski space, the Lagrangian reads

$$
\mathcal{L}_{h}=\frac{1}{2}\left(D_{\mu} \Phi\right)^{\dagger}\left(D^{\mu} \Phi\right)-\frac{1}{4} H_{\mu \nu} H^{\mu \nu}-V(\Phi),
$$

where $H_{\mu \nu}$ is the field strength tensor associated with the vector gauge boson $B_{\mu}$ and

$$
D_{\mu}=\partial_{\mu}+i g B_{\mu}
$$

At high enough temperature, loop corrections from the thermal bath restore the $U(1)$ symmetry and the effective potential has an overall minimum at $|\Phi|=0[1,59]$. Starting from high enough temperature, one therefore expects the $U(1)$ symmetry to be spontaneously broken during the expansion and cooling of the universe. During the phase transition, the Higgs field reaches its new vacuum expectation value $\Phi=\eta_{\nu} \mathrm{e}^{i \theta}$. At each spacetime location, the phase $\theta\left(x^{\mu}\right)$ will have a given value, all of them being uncorrelated on distances larger than the typical correlation length of the phase transition. As pointed by Kibble, this is at most the horizon size $d_{\mathrm{h}} \propto t$ although one expects it to be much smaller $[3,60-63]$. As a result, there exists closed paths in space along which $\theta$ varies from 0 to $2 \pi$ (or a multiple of $2 \pi$ ). Such phase configurations necessarily encompass a point at which $|\Phi|=0$ (see Figure 1); the old vacuum has been trapped into a nontrivial configuration of the new vacuum, and this prevents its decay. Such a structure is invariant by translations along the third spatial dimension and is string shaped.

Solitonic solutions of the field equations describing a static straight Abelian string can easily be computed under the Nielsen-Olesen ansatz. The transverse profile of the Higgs and gauge field are assumed to be [64]

$$
\Phi=\eta_{\mathrm{v}} H(\varrho) \mathrm{e}^{i n \theta}, \quad B_{\mu}=\frac{Q(\varrho)-n}{g} \delta_{\mu \theta},
$$

where $(r, \theta)$ stands for a polar coordinate system aligned along the string. The dimensionless radial coordinate has been defined by $\varrho=m_{h} r$ where, $m_{\mathrm{h}}=\sqrt{\lambda} \eta_{\mathrm{v}}$ is the mass of the Higgs boson. The integer $n$ is the "winding number" and gives the number of times the Higgs winds the potential for one rotation around the string. From (2), the dimensionless equations of motion read

$$
\begin{gathered}
\frac{\mathrm{d}^{2} H}{\mathrm{~d} \varrho^{2}}+\frac{1}{\varrho} \frac{\mathrm{d} H}{\mathrm{~d} \varrho}=\frac{H Q^{2}}{\varrho^{2}}+\frac{1}{2} H\left(H^{2}-1\right), \\
\frac{\mathrm{d}^{2} Q}{\varrho^{2}}-\frac{1}{\varrho} \frac{\mathrm{d} Q}{\mathrm{~d} \varrho}=\frac{m_{b}^{2}}{m_{h}^{2}} H^{2} Q,
\end{gathered}
$$

where $m_{b}=g \eta_{v}$ is the mass of the vector gauge boson. In Figure 2, we have represented the string solution to these equations in Minkowski spacetime [65, 66]. The boundary conditions are such that the Higgs field vanishes at the center of the string to reach its vacuum expectation value (vev) asymptotically. This typically happens after a length scale given by its Compton wavelength $1 / m_{h}$. Similarly, the gauge field boundary conditions are such that it has vanishing derivative in the core and remains finite far from the string. As shown in Figure 2, it actually condenses inside the string with a spatial extension roughly equal to $1 / m_{b}$.

The energy content of such a string is given by the stress tensor stemming from the Lagrangian of (2). Along the string worldsheet,

$$
\begin{aligned}
T^{t t} & =-T^{z z} \\
& =\frac{\lambda \eta_{\mathrm{v}}^{4}}{2}\left[\left(\partial_{\varrho} H\right)^{2}+\frac{Q^{2} H^{2}}{\varrho^{2}}+\frac{\left(H^{2}-1\right)^{2}}{4}+\frac{\lambda}{g^{2}} \frac{\left(\partial_{\varrho} Q\right)^{2}}{\varrho^{2}}\right],
\end{aligned}
$$

which are the only two components which do not vanish after an integration over the transverse coordinates. Integrating the temporal part gives the string energy per unit length $U$, whereas the longitudinal component gives $-T$. One finally gets

$$
U=T=C\left(\frac{\lambda}{g^{2}}\right) \eta_{v}^{2}
$$




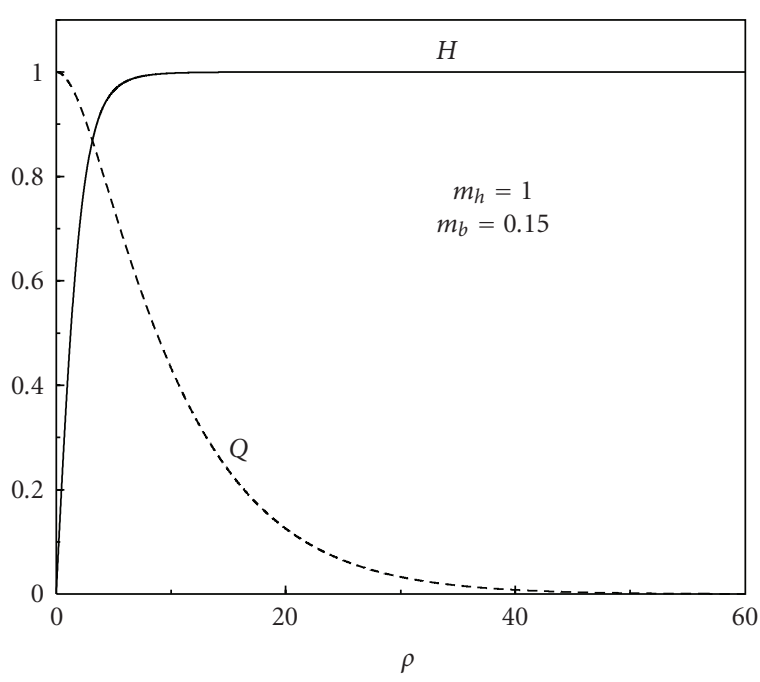

Figure 2: String forming field profiles in the Abelian Higgs model with unity winding number. The Higgs field $H$ vanishes in the vortex core and reaches its vacuum expectation value within distances given by the Higgs Compton wavelength $1 / m_{h}$. The gauge field $Q$ condenses inside the vortex over distances given by $1 / m_{b}$.

where $C\left(\lambda / g^{2}\right)$ is an order unity function at fixed winding number. Increasing the winding number centrifuges the energy density around the core such that $U$ is changed in a more complex way [5]. This immediately shows that cosmic strings generically carry an energy density and tension of the order of the symmetry breaking energy scale $U \simeq \eta_{\mathrm{v}}^{2}$. Notice that along the string direction the pressure $P_{z}=-T=$ $-U$ is negative, and we are in presence of a "cosmological constant wire", as one may expect from a Lorentz invariant vacuum object. Consequently, the trace of the stress tensor $\eta_{\mu \nu} T^{\mu \nu}$ vanishes and cosmic strings do not induce any Newtonian gravitational potential. Together with the socalled cosmological scaling behaviour (see below), this is the mere reason why they remain cosmologically acceptable. They do however induce dynamical gravitational effects, the metric far from the string core being Minkowski with a missing angle [67] (see Section 4).

The Abelian string model is intensively used in the literature to explore the string forming phase transition and string interactions $[68,69]$. From the Kibble's argument, one expects the phase of the Higgs field to be random and the resulting string path should be a self-avoiding random walk with a given correlation length [70]. Performing lattice simulations allows to probe in more details the string forming mechanism and gives a more accurate picture of a cosmic string network just after its formation [71-74]. Abelian Higgs simulations are also used to compute the cosmological evolution of such a network [75-77] (see Section 3).

\subsection{Other Flux Tubes}

Global String. The Abelian string provides an explicit example of the formation of line-like topological defects by the spontaneous breakdown of a gauged symmetry. Breaking a $U(1)$ global symmetry can also produce topological defects, the so-called global strings. However, in the absence of gauge fields, one can show that global cosmic strings exhibit long-range interactions and Goldstone radiation [78]. Their dynamics can however mimic local strings and being cosmologically acceptable in some regime [79-81].

Non-Abelian String. If the broken symmetry group $G$ is non-Abelian, the cosmic strings formed during the phase transition exhibit new properties compared to the $U(1)$ kind [5]. In particular, the mapping of the Higgs field to the real space can be made along different broken generators $\Phi_{a}=$ $\eta_{v} \exp \left(i T_{a} \theta\right)$. This implies that different type of non-Abelian strings may be formed and will interact with each others according to their respective windings. The classic example being the appearance of a $\left[T_{a}, T_{b}\right]$-string from the crossing between a $T_{a}$-string and $T_{b}$-string [82]. In the cosmological framework, new strings can potentially be formed at each interaction leading to a frustrated intricate configuration [83-86]. Such an outcome depends on the underlying nonAbelian gauge group and Abelian string-like evolution can also be recovered, as for instance in the $U(N)$ models [8791].

Semilocal String. String-shaped energy density distribution can also appear even if the vacuum manifold is simply connected. The nontrivial topology argument is indeed only a sufficient condition of defect appearance. The electroweak symmetry breaking scheme enters this class, although the first homotopy group is trivial, semilocal strings can be formed $[92,93]$. A simple description of these strings can be obtained by replacing the Higgs field in the Abelian model by a doublet in a $S U(2)$ global representation [94]. These flux tube configurations are stabilised because they can be energetically favoured for some values of the model parameters, typically for $m_{b}>m_{h}$ [95]. Let us notice that the currently measured electroweak model parameters do not support stable vortices [94]. Similar vortices could also be formed during a chiral symmetry breaking phase transition [96-103].

$K$ - and DBI-String. These are another extensions of the Abelian Higgs string for which the scalar and gauge field kinetic terms are noncanonical, or of the Dirac-Born-Infeld form [104-107]. These strings essentially differ from their Abelian counterparts when the gradient terms are nonvanishing, that is, in the core.

Current Carrying String. In minimal extensions of the Abelian Higgs model, one may couple extra-scalar fields to the string forming Higgs field. As shown by Witten, this can lead to the condensation of the extrascalar over the string core [108]. The resulting string is carrying a current that breaks the longitudinal Lorentz invariance; $U$ and $T$ are no longer degenerated and the string dynamics is affected $[38,65,109]$. One of the most important consequence of these currents is the potential appearance of centrifugally 
supported loops. If stable, these so-called vortons could efficiently populate the universe and avoiding the overclosure gives strong constraints on the cosmic string energy scale $[110,111]$. A similar mechanism works for the fermionic fields which are Yukawa coupled to the string forming Higgs field. They generically produce currents along the string with a discrete mass spectrum, in a way similar to the photon propagation in waveguides [112]. Unless the massive propagation modes are not excited, the resulting loops are however expected to be unstable $[113,114]$.

2.3. Cosmic Superstrings. Cosmic superstrings are fundamental line-shaped objects that can be formed at the end of brane-inflation (see $[34-36,115,116]$ for reviews). The idea that fundamental quantum strings can be stretched to cosmological distances has been mentioned by Witten [117]. If stable, one would expect fundamental strings to be at an energy scale close to the String Theory scale, that is, close to the Planck mass, and this is trivially ruled out by observations. In addition, current $\mathrm{CMB}$ constraints tell us that the energy scale of inflation is at most the GUT scale [118], implying that strings formed at a higher energy would have been diluted anyway. The situation changed with the discovery that inflation within String Theory could be a geometrical phenomena induced by the motion of a brane moving in a warped throat, somewhere in the compact manifold of the extra-dimensions [26]. In the KKLMMT model $[28,119]$, the inflaton is a scalar degree of freedom associated with the position $r$ of a D3 brane in a warped throat. Within a ten-dimensional super-gravity ansatz for the metric, in the type IIB String Theory,

$$
\mathrm{d} s^{2}=\frac{1}{\sqrt{h(r)}} g_{\mu \nu} \mathrm{d} x^{\mu} \mathrm{d} x^{\nu}+\sqrt{h(r)}\left(\mathrm{d} r^{2}+r^{2} \mathrm{~d}_{5} s^{2}\right),
$$

and the throat is described by the warping function $h(r)$ (explicitly, it can be the Klebanov-Strassler conifold [120]). In this system, accelerated expansion of our universe comes from the interaction of this brane with an anti-D3 brane sitting at the bottom of the throat $r_{0}$. Current CMB data suggest that inflation preferentially ends by violation of the slow-roll conditions, whereas the system continues to evolve till the two branes collide [121]. The brane interactions at that stage require String Theory calculations and are expected to trigger a reheating era accompanied by a copious production of various D1-branes and fundamental F-strings [29-32]. Since the brane annihilation takes place at the bottom of the throat, due to the warped metric, the cosmic superstring tensions measured by an exterior fourdimensional observer are redshifted by a factor $h^{1 / 2}\left(r_{0}\right)$. The resulting effect is to significantly lower the string tension down to acceptable values. In fact, the stability of the produced $\mathrm{F}$-strings and $\mathrm{D}$-strings require additional constraints on the model parameters and the spectrum of superstring tensions depending on the underlying scenario [32]. For instance, in the KKLMMT model, one expects $10^{-10}<G U<10^{-7}[122]$.

Cosmic superstrings differ from the Abelian strings in various aspects. In addition to the coexistence of two different types, they can form bound states of $p$ F-strings and $q \mathrm{D}$-strings. The tension of these $(p, q)$-strings depends on $p, q$, the binding energy but also on their configuration in the throat $[123,124]$. In fact, many of $(p, q)$-string properties mimic the non-Abelian type of topological vortex, as the existence of bound states and Y-junctions [125128]. Such similarities have actually been used to probe the properties of the cosmic superstrings through the more tractable framework of field theory [129-132].

2.4. Infinitely Thin Strings. These are the one-dimensional version of the relativistic point particles. Following Carter macroscopic covariant approach [38, 109, 133, 134], string events can be localised in the four-dimensional spacetime by the so-called embedding functions $x^{\mu}=X^{\mu}\left(\xi^{a}\right)$, where $\xi^{0}$ and $\xi^{1}$ are a timelike and spacelike internal coordinate of the string worldsheet. Denoting by $g$ the four-dimensional metric tensor, one can define the two-dimensional induced metric

$$
\gamma_{a b}=g_{\mu \nu} \frac{\partial X^{\mu}}{\partial \xi^{a}} \frac{\partial X^{\nu}}{\partial \xi^{b}}
$$

such that the infinitesimal interval between two events reduces to $\mathrm{d} s^{2}=\gamma_{a b} \mathrm{~d} \xi^{a} \mathrm{~d} \xi^{b}$. From its inverse, on can define the first fundamental tensor

$$
q^{\mu \nu}=\gamma^{a b} \frac{\partial X^{\mu}}{\partial \xi^{a}} \frac{\partial X^{v}}{\partial \xi^{b}}
$$

which is nothing but a projector over the string worldsheet. Similarly, $\perp_{\nu}^{\mu} \equiv g_{\nu}^{\mu}-q_{\nu}^{\mu}$ is an orthogonal projector and they verify

$$
q_{\rho}^{\mu} q_{\nu}^{\rho}=q_{\nu}^{\mu}, \quad \perp_{\rho}^{\mu} \perp_{\nu}^{\rho}=\perp_{\nu}^{\mu}, \quad q_{\rho}^{\mu} \perp_{\nu}^{\rho}=0 .
$$

Variations of the first fundamental form are encoded in the second fundamental tensor

$$
K_{\mu \nu}^{\rho} \equiv q_{\nu}^{\alpha} \bar{\nabla}_{\mu} q_{\alpha}^{\rho},
$$

where a bar quantity stands for the projection of its fourdimensional analogue over the worldsheet, that is, $\bar{\nabla}_{\mu} \equiv$ $q_{\mu}^{\alpha} \nabla_{\alpha}$. Integrability imposes $K_{[\mu \nu]}^{\rho}=0$ and, by construction, the second fundamental form is, respectively, tangent and orthogonal to the worldsheet on its first and last indices. As a result, contracting the first two tangential indices gives a purely orthogonal vector which measures the string extrinsic curvature [134]

$$
K^{\rho} \equiv K_{\mu}^{\mu \rho}=\bar{\nabla}_{\mu} q^{\mu \rho} .
$$

The energy content of a spacetime two-dimensional surface can be characterised by its internal stress energy tensor. Similarly to the cosmological perfect fluid, one may consider a string whose stress-energy tensor is diagonal in a preferred basis. Positivity of the energy conditions ensures that the timelike eigenvalue $U>0$, while the spacelike eigenvalue $T$ should verify $|T| \leq U[135]$. In this frame, $U$ represents the energy per unit length of the string and $T$ the 
string tension. Denoting by $u^{\mu}$ and $v^{\nu}$ the respective timelike and spacelike orthonormal eigenvectors, one has

$$
\bar{T}^{\mu \nu}=U u^{\mu} u^{\nu}-T v^{\mu} v^{\nu}=(U-T) u^{\mu} u^{\nu}-T q^{\mu \nu},
$$

where

$$
\begin{gathered}
u^{\alpha} u_{\alpha}=-1, \quad v^{\alpha} v_{\alpha}=1, \quad u^{\alpha} v_{\alpha}=0, \\
q^{\mu \nu}=-u^{\mu} u^{\nu}+v^{\mu} v^{\nu} .
\end{gathered}
$$

In the absence of external forces, reparametrisation invariance of the string worldsheet ensures the stress-energy pseudo-conservation from Noether's theorem [136]

$$
\bar{\nabla}_{\rho} \bar{T}^{\rho \sigma}=0 .
$$

As for a cosmological fluid, these equations are not sufficient to close the equations of motion for the string. One has to supplement them by an equation of state of the fluid under scrutiny. The simplest case is the so-called barotropic model for which the equation of state is the relation $U(T)$. One can then introduce the two Legendre conjugated parameters

$$
\ln \bar{\nu}=\int \frac{\mathrm{d} U}{U-T}, \quad \ln \bar{\mu}=\int \frac{\mathrm{d} T}{T-U},
$$

such that $U-T=\overline{\mu \nu}$. Clearly, $\bar{\nu}$ plays the role of a number density and its Legendre conjugated parameter $\bar{\mu}$ will therefore be a chemical potential, that is, an effective mass carried per unit number density. Defining their respective worldsheet current density by

$$
\bar{\mu}^{\rho} \equiv \bar{\mu} u^{\rho}, \quad \bar{\nu}^{\rho} \equiv \bar{v} u^{\rho},
$$

one can rewrite (16) as

$$
\bar{\nabla}_{\rho} \bar{T}_{\sigma}^{\rho}=\bar{\mu}_{\sigma} \bar{\nabla}_{\rho} \nu^{\rho}+\bar{\nu}^{\rho} \bar{\nabla}_{\rho} \bar{\mu}_{\sigma}-\bar{\nu}^{\rho} \bar{\nabla}_{\sigma} \bar{\mu}_{\rho}-\mathrm{T} K_{\sigma}=0 .
$$

Contracting (19) with $\bar{\nu}^{\sigma}$ ensures the current conservation along the string

$$
\bar{\nabla} \rho \bar{\nu}^{\rho}=0,
$$

while its projection onto the worldsheet gives the momentum transport law

$$
q_{\alpha}^{\sigma} u^{\rho} \bar{\nabla}_{[\rho} \bar{\mu}_{\sigma]}=0 .
$$

Finally, the orthogonal projection of (19) reduces to

$$
K^{\rho}=\perp_{\sigma}^{\rho} \frac{\bar{\nu}^{\alpha} \bar{\nabla}_{\alpha} \bar{\mu}^{\sigma}}{T}=\perp_{\sigma}^{\rho}\left(\frac{U}{T}-1\right) \dot{u}^{\sigma},
$$

where the string acceleration $\dot{u}^{\sigma}$ stands for

$$
\dot{u}^{\sigma} \equiv u^{\alpha} \bar{\nabla}_{\alpha} u^{\sigma} .
$$

As should be clear from (14), the barotropic equation of state breaks Lorentz invariance along the string for $U \neq T$. In fact, it describes a wide class of elastic string models [137-139], and as suggested by (20), the scalar current carrying cosmic strings [140]. Conversely, imposing Lorentz invariance along the worldsheet reduces the equation of state to the trivial form $U=T$ (see (14)), which is also the relation found for the Abelian Higgs string. This infinitely thin string is the Nambu-Goto (NG) string and does not possess any internal structure [141]. The associated equations of motion are purely geometrical and do not depend on $U$. From (22), they reduce to the vanishing of the extrinsic curvature vector, that is,

$$
K^{\rho}=0,
$$

which can be rewritten in a coordinate-dependant way by using(13)

$$
K^{\mu}=\frac{1}{\sqrt{-\gamma}} \partial_{a}\left(\sqrt{-\gamma} \gamma^{a b} \partial_{b} X^{\mu}\right)+\Gamma_{\nu \rho}^{\mu} \gamma^{a b} \partial_{a} X^{\nu} \partial_{b} X^{\rho} .
$$

The connections $\Gamma_{\nu \rho}^{\mu}$ are for the background spacetime of metric $g_{\mu \nu}$ while $\gamma$ is the determinant of the induced metric. These equations can also be recovered from the usual NG action with an explicit coordinate system [5]

$$
S=-U \int \mathrm{d}^{2} \xi \sqrt{-\gamma} .
$$

\section{Cosmological Evolution of Nambu-Goto Strings}

The previous section shows that the equations of motion of an isolated string depend on the underlying microscopic model. The type of string is more determinant when two strings interact; cosmic superstrings may form bound states, while non-Abelian vortices may weave new vortices from each of their interaction points. Understanding the cosmological evolution of a string network requires one to solve both the local equations of motion for each string and the outcome of their interactions when they meet. Moreover, the evolution of a system of strings starts from an initial configuration which should describe the network configuration just after its formation. Numerical simulations have been used to overcome some of the abovementioned difficulties and, up to now, Friedmann-LemaîtreRobertson-Walker (FLRW) network simulations have only been performed with Nambu-Goto strings, Abelian Higgs strings and semi-local strings [75-77, 142-149], up to some variations $[80,81,131,150]$. As a result, extrapolating the following results to other types of string should be made with caution. On the bright side, (22) suggests that as long as the string acceleration remains small compared to $T /(U-T)$, one expects the equations of motion of the string to be close to the NG case (up to the eventual vortons appearance). In the following, we describe the results obtained for NG strings. Some differences exist with the results obtained in the Abelian Higgs string simulations.

Before entering into details, let us summarize two fundamental properties these simulations have revealed. The first is that a cosmic string network avoids cosmological domination by evacuating most of its excess energy through some complex mechanisms, which typically result in transferring energy between the horizon-sized distances 
and the smaller length scales. For NG simulations, this is the formation of cosmic string loops whereas in Abelian Higgs simulation boson radiation is involved. The second property is that the influence of the initial conditions is expected to disappear on the length scales of astrophysical interests. A network of cosmic strings relaxes towards a cosmological attractor which depends only on the expansion rate; this is the so-called scaling regime.

3.1. Dynamics. The equations of motion for NG strings are the vanishing of the extrinsic curvature vector $K^{\mu}=0$. In a flat FLRW background,

$$
\mathrm{d} s^{2}=a^{2}(\eta)\left(-\mathrm{d} \eta^{2}+\delta_{i j} \mathrm{~d} x^{i} \mathrm{~d} x^{j}\right),
$$

Equation (25) can be simplified with the transverse gauge fixing conditions

$$
g_{\mu \nu} \frac{\partial X^{\mu}}{\partial \tau} \frac{\partial X^{\nu}}{\partial \sigma}=0
$$

with the notation $\tau=\xi^{0}$ and $\sigma=\xi^{1}$ for the timelike and spacelike string coordinates. Such a choice of coordinates reflects the property that an NG string is Lorentz invariant along the worldsheet; there is no physical longitudinal component of the string velocity. In this gauge, the equations of motion read

$$
\begin{aligned}
\ddot{X}^{\mu} & +\left(\frac{\dot{\varepsilon}}{\varepsilon}+\frac{2}{a} \frac{\mathrm{d} a}{\mathrm{~d} X^{0}} \dot{X}^{0}\right) \dot{X}^{\mu}-\frac{1}{\varepsilon}\left(\frac{X^{\prime}}{\varepsilon}\right)^{\prime} \\
& -\frac{2}{a} \frac{\mathrm{d} a}{\mathrm{~d} X^{0}} \frac{X^{0}}{\varepsilon} \frac{X^{\prime}}{\varepsilon}+\delta_{0}^{\mu} \frac{2}{a} \frac{\mathrm{d} a}{\mathrm{~d} X^{0}} \dot{X}^{2}=0,
\end{aligned}
$$

where a "dot" and a "prime" stand, respectively, for differentiation with respect to $\tau$ and $\sigma$. We have also defined the quantity

$$
\varepsilon \equiv \sqrt{-\frac{X^{\prime}}{\dot{X}^{2}}}
$$

The conditions in (28) do not completely fix the coordinate degrees of freedom and one can supplement them with the so-called temporal gauge fixing which identifies the timelike coordinate with the background time at the string event: $\tau=$ $X^{0}=\eta$. In the transverse temporal gauge, $(28)$ reads $\dot{\vec{X}} \cdot \stackrel{\prime}{\vec{X}}=$ 0 , while (29) simplifies to

$$
\ddot{\vec{X}}+2 \mathscr{H}\left(1-\dot{\vec{X}}^{2}\right)-\frac{1}{\varepsilon}\left(\frac{\stackrel{\vec{X}}{X}_{\varepsilon}}{\varepsilon}\right)^{\prime}=0, \quad \dot{\varepsilon}+2 \mathscr{H} \varepsilon \dot{\vec{X}}^{2}=0,
$$

with

$$
\varepsilon=\sqrt{\frac{{\frac{\prime^{2}}{X}}_{1-\dot{\vec{X}}^{2}}^{2}}{}}
$$

and $\mathscr{H}$ is the conformal Hubble parameter. The vector symbols being understood as three-dimensional spatial vectors. Numerically, it is much more convenient to solve an equivalent set of equations found by Bennett and Bouchet [144]. Defining the new vectors $\vec{p}$ and $\vec{q}$ as

$$
\vec{p}(\tau, u) \equiv \frac{\stackrel{\prime}{X}}{\varepsilon}-\dot{\vec{X}}, \quad \vec{q}(\tau, v) \equiv \frac{\stackrel{\prime}{\vec{X}}}{\varepsilon}+\dot{\vec{X}}
$$

evaluated at the new coordinates $u=\int \varepsilon \mathrm{d} \sigma-\tau$ and $v=$ $\int \varepsilon \mathrm{d} \sigma+\tau$, the equations of motion (31) can be recast into

$$
\begin{gathered}
\frac{\partial \vec{p}}{\partial \tau}=-\mathscr{H}[\vec{q}-\vec{p}(\vec{p} \cdot \vec{q})], \\
\frac{\partial \vec{q}}{\partial \tau}=-\mathscr{H}[\vec{p}-\vec{q}(\vec{p} \cdot \vec{q})], \\
\frac{\dot{\varepsilon}}{\varepsilon}=-\mathscr{H}(1-\vec{p} \cdot \vec{q}) .
\end{gathered}
$$

As an illustrative example, these equations have an exact solution in Minkowski space. Taking $\mathscr{H}=0$, one immediately gets $\varepsilon=1$ (up to a normalisation constant), $\vec{p}(u)$ and $\vec{q}(v)$ are constant over the characteristics $u=\sigma-\tau$ and $v=\sigma+\tau$. Inverting (33) gives

$$
\stackrel{\prime}{X}(\tau, \sigma)=\frac{1}{2}[\vec{p}(\sigma+\tau)+\vec{q}(\sigma-\tau)]
$$

which describes the propagation of left and right moving string deformations at the speed of light. In the FLRW background, these modes are no longer free moving, but interact through the Hubble term (see (34)). Solving these equations gives the $X^{\mu}(\tau, \sigma)$ for each strings but does not predict what happens when two strings collide.

3.2. Collisions. In the infinitely thin approach, the outcome of an NG string intersection event cannot be predicted. Stress tensor conservation equations require either that the two strings pass through each others, or they intercommute as sketched in Figure 3. The outcome of a string collision process can only be addressed within the framework of a microscopic model. Numerical simulations of interactions have been performed for a variety of models, and in particular for the Abelian Higgs string as represented in Figure 4. In this case, unless the relative string velocity is close to unity $[151,152]$, or the strings are almost parallel, string intercommutation generically occurs $[153,154]$. Let us notice that for type I Abelian strings (i.e., having $m_{\mathrm{b}}>m_{\mathrm{h}}$ ), bound states of the two strings can also be formed at low velocity [155-157].

The situation is not necessarily the same for the other types of string. As already mentioned, non-Abelian strings can weave new strings from their intersection points while current carrying cosmic strings intercommute for bosonic carriers [158]. In the case of cosmic superstrings, it has been shown that they intercommute with a probability depending on the fundamental string coupling, a quantity 


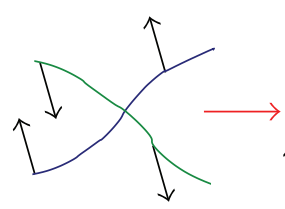

(a)

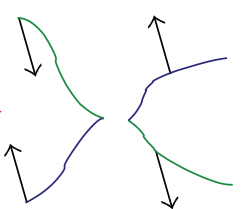

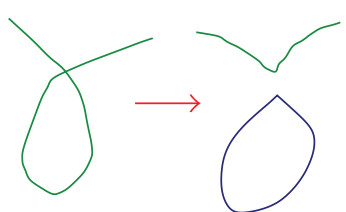

(b)

FIGURE 3: Intercommuting strings exchanging their partners (a). On (b), the same mechanism produces a loop from a self-intercommutation. The arrows represent velocity vectors.

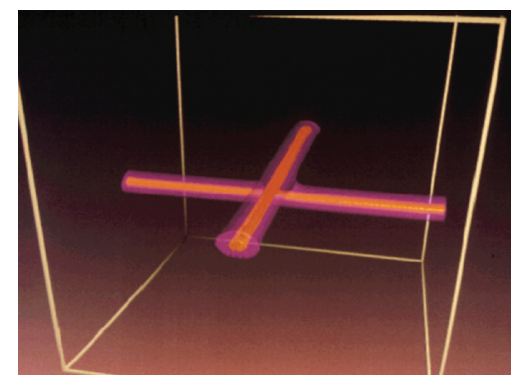

(a)

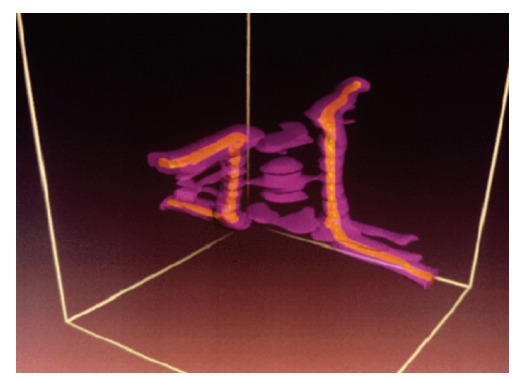

(c)

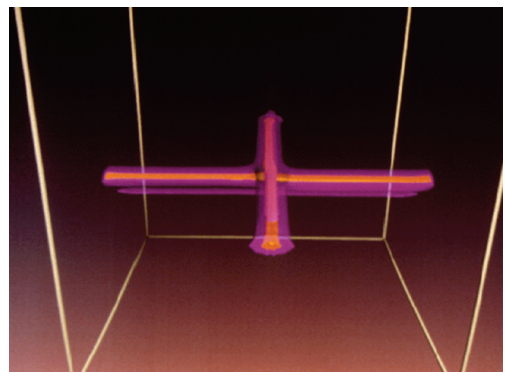

(b)

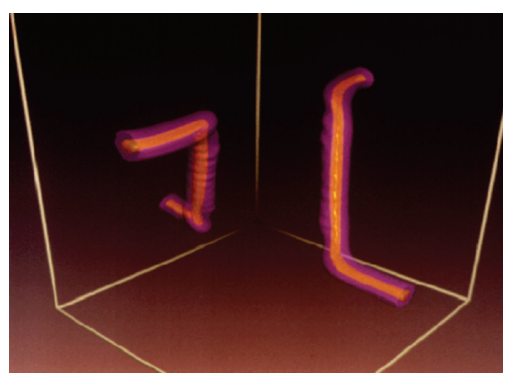

(d)

Figure 4: Numerical simulations of the intersection between two Abelian Higgs strings [154]. The Higgs and gauge field profiles are represented as the inner orange and outer pink tubes. Generically, Abelian Higgs strings with $m_{h}>m_{b}$ intercommute.

which can be significantly smaller than unity [159]. In the case of $(p, q)$-string collisions, Y-junctions can be formed under some kinematic constraints [126, 157, 160-163]. Concerning NG simulations, string collisions are actually implemented through a phenomenological probability $P_{e}$ of intercommutation at each intersection event.

3.3. Initial Conditions. Solving the cosmological evolution of a NG string network amounts to solving (34) and (35) along each string, finding all of their intersection points and implementing an intercommutation, or not, with the probability $P_{e}$. The network evolution is now uniquely determined once the initial conditions are specified. The simplest way to set initial conditions is through the Vachaspati-Vilenkin (VV) algorithm [164]. Motivated by the Kibble mechanism, one assumes a $U(1)$ Higgs field to be uncorrelated above a given correlation length $\ell_{c}$. A cosmic string will cross a given plane if one can find a closed loop along which its phase runs from 0 to a multiple of $2 \pi$. On a discrete three-dimensional lattice, of $\ell_{c}$-spacing, it is sufficient to approximate $U(1)$ by $Z_{3}$ and randomly choose the phase at each corner from three values $0,2 \pi / 3$ and $4 \pi / 3$ to decide if a string crosses the associated face. Other symmetry breaking schemes and lattice can be approximated in a similar way [165-169]. In Figure 5, we have shown the initial string network configuration obtained from the VV algorithm. The string paths have been smoothed by replacing the right angles by circles of radius $\ell_{c}$. The initial network configuration obviously depends on the physical parameter $\ell_{c}$, the network correlation length. In FLRW spacetime, there is, however, another physical parameter which has to be specified; the distance to the horizon $d_{h}$. From those, the initial string energy density is now uniquely determined. A random transverse velocity field can also be added along each string since one does not expect the strings to be initially at rest in any realistic setup. At this point, let us mention that the numerical implementation of the VV initial conditions introduce two additional purely numerical parameters: the size of the periodic box which contains the simulation, usually normalised to unity in comoving coordinates, and the discretisation step required to represent a string, usually given by $N_{\text {ppcl }}$, the number of points per correlation length. 


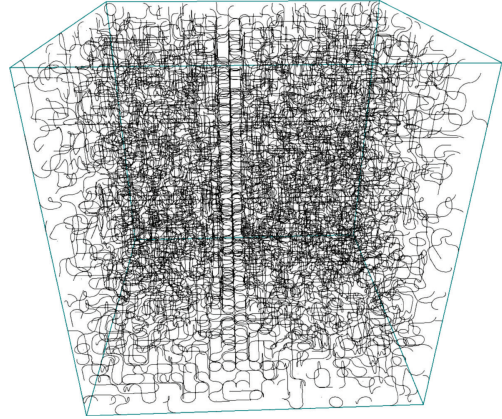

FIGURE 5: Initial string network configuration from the VachaspatiVilenkin algorithm. The box is a small lattice of $25 \ell_{c}$ for illustration purpose only.

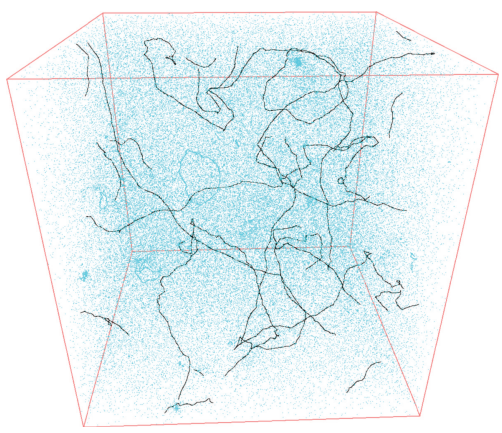

FIgURE 6: String network configuration in the matter era when the horizon fills the whole simulation box. String loops with a length smaller than the distance to the horizon appear in blue.

\subsection{Cosmological Scaling}

3.4.1. Long Strings. By switching on the evolution from the initial network, string motion and intersections drastically change the shape of the strings as well as the network aspect (see Figure 6). Naively, without any collisional process, one would expect the string network to dominate the energy density of the universe. In a volume $V$, denoting by $\ell_{\infty}$ the typical correlation length of the network at a given time (initially $\ell_{\infty}=\ell_{c}$ ), the number of strings should be roughly given by $V / \ell_{\infty}^{3}$. The resulting energy density should therefore be

$$
\rho_{\infty} \simeq \frac{V}{\ell_{\infty}^{3}} \times\left(U \ell_{\infty}\right) \times \frac{1}{V}=\frac{U}{\ell_{\infty}^{2}} .
$$

Due to cosmological expansion one has $\ell_{\infty} \propto a$ and $\rho_{\infty} \propto$ $1 / a^{2}$. As noted by Kibble, this domination does not occur due to intercommutation processes which allow the formation of loops. In the so-called "one scale model", Kibble [3] assumes that loops of typical size $\ell_{\infty}$ are formed at a rate equals to $\ell_{\infty}^{-4}$ (for relativistic speeds, one expects one intercommutation per string during the time $\ell_{\infty}$ ). As a result, during a time interval $\delta t$, the energy density transferred to loops is

$$
\delta \rho_{\infty \rightarrow 0} \simeq \ell_{\infty}^{-4} \delta t U \ell_{\infty} .
$$

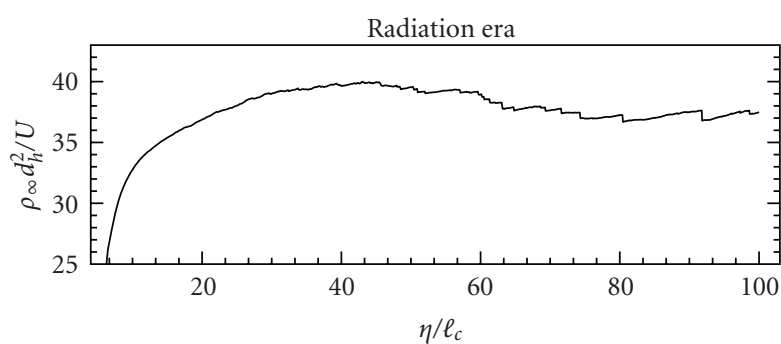

(a)

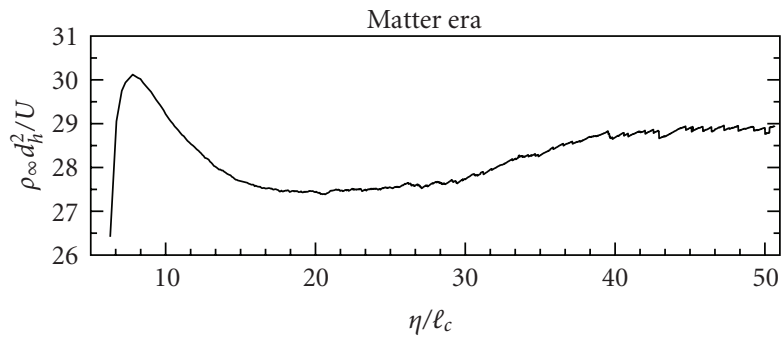

(b)

Figure 7: Energy density of super-horizon sized strings as a function of the conformal time (in unit of $\ell_{c}$ ) in the radiation and matter era. After some relaxation time, $\rho_{\infty} \propto 1 / d_{h}^{2}$, the proportionality factor is universal.

From (37), the energy density of strings which are not loops verifies

$$
\frac{\mathrm{d} \rho_{\infty}}{\mathrm{d} t} \simeq-2 H \rho_{\infty}-\frac{\rho_{\infty}}{\ell_{\infty}}
$$

where $\ell_{\infty}$ is a function of the cosmic time. Defining $C(t)=$ $\ell_{\infty}(t) / t$, this equation can be recast into

$$
\frac{1}{C} \frac{\mathrm{d} C}{\mathrm{~d} t} \simeq-\frac{1}{2 t}\left(\frac{2+6 w}{3+3 w}-\frac{1}{C}\right),
$$

where the background cosmological fluid sourcing the universe expansion has an equation of state $P=w \rho$. The constant solution $C(t)=(3+3 w) /(2+6 w)$ is an attractor for which

$$
\rho_{\infty} \propto \frac{U}{t^{2}} \propto \frac{U}{a^{3(1+w)}} .
$$

The energy density associated with strings which are not loops "scales" as matter in the matter era and radiation in the radiation era. Therefore, it is prevented to dominate over the usual cosmological fluids and cannot overclose the universe. Notice however that the total energy density could still dominate the cosmological dynamics if the energy density under the form of loops is not evacuated by some extramechanism. For NG cosmic strings, loops are transformed into radiation due to the emission of gravitational waves [170173]. Other types of loops may lose energy by different radiative processes, such as particle emission, or even energy leakage into the extra-dimensions in the case of cosmic superstrings [174, 175]. In Figure 6, we have represented an evolved string network at the end of a matter era run. 
For FLRW simulations within a fixed comoving box with periodic boundary conditions, one cannot evolve the system indefinitely; at some point, periodic boundaries become causally connected. Usually, one stops the run when the distance to the horizon fills the simulation volume, or more rigorously half of it. In this figure, one sees that only a few super-horizon strings remain (black long strings) whereas the box is also filled with a lot of small loops (blue) and a few larger loops having a size typical of distance between two long strings. The latter are freshly formed Kibble loops whereas the existence of the small ones cannot be explained in the framework of the one scale model [144]. Concerning the energy density associated with the super-horizon strings (also called infinite strings), their evolution in the matter and radiation era have been plotted in Figure 7; they "scale" as expected. From [146], one has

$$
\left.\rho_{\infty} \frac{d_{h}^{2}}{U}\right|_{\mathrm{mat}}=28.4 \pm 0.9,\left.\quad \rho_{\infty} \frac{d_{h}^{2}}{U}\right|_{\mathrm{rad}}=37.8 \pm 1.7 .
$$

As the behaviour of the energy density associated with long strings suggests, the time evolution drives the string network towards a stable cosmological configuration which does not seem to depend on its initial configuration, at least for the long strings. Figure 6, therefore, displays what a cosmological string network should look like inside a horizon volume, at any time during the matter era. As is clear from Figure 7, the relaxation time required for the energy density of long strings to reach the attractor is small. Concerning the cosmic string loops, their existence and behaviour have been the subject of various claims and analytical works [146, 148, 176$181]$. In the following, we present recent results $[146,182]$ showing that the energy density of loops also reaches a scaling evolution similar to (42).

3.4.2. Loops. As previously mentioned, the small loops observed in NG simulation cannot be explained in the framework of the one scale model. These loops find their origin from the self-intercommutation of strings on length scales typical of their small size. The building of a small scale structure on strings is the outcome of the successive intersection events during which new kinks are produced and propagate along the intercommuted segments. Correlations between the kinks induce, from (33), auto- and crosscorrelations between $\dot{\vec{X}}\left(\sigma_{1}\right)$ and $\stackrel{\prime}{\vec{X}}\left(\sigma_{2}\right)$ from which small loops can be produced $[176,179]$. In Figure 8 , we have plotted the energy density distribution under the form of loops with respect to the conformal time during the radiation and matter era. The simulation performed is one of the largest up to date; the box contains $100 \ell_{\mathrm{c}}$ whereas the redshift simulation range reaches almost two orders of magnitude. The loop energy density distribution $\mathrm{d} \rho_{\circ} / \mathrm{d} \alpha$ is defined such that $\mathrm{d} \rho_{\circ}(\alpha)$ is the energy density carried by all loops having a physical length $\ell$ in the range $\alpha d_{h}$ to $(\alpha+\mathrm{d} \alpha) d_{h}$. In other words, we measure loop size in unit of the horizon length. (This is the relevant physical length scale of the problem.) A logarithmic binning in $\alpha$ of resolution $\Delta \alpha / \alpha \simeq 10^{-1}$ has been used in the range $\left[10^{-5}, 10^{2}\right]$ to compute these quantities. From this plot, it is clear that after an overproduction regime characterized by the bump of Figure 8, the energy density distribution of loops of given size $\alpha$ relaxes towards a stationary regime in which it scales as $1 / d_{h}^{2}$. Such an observation implies that, once relaxed, the loop number density distribution is of the form

$$
\frac{\mathrm{d} n}{\mathrm{~d} \alpha}=\frac{\wp(\alpha)}{\alpha d_{\mathrm{h}}^{3}},
$$

where the "scaling function" $\delta(\alpha)$ is found to be well fitted by the power laws [146] $\delta(\alpha)=C_{\circ} \alpha^{-p}$ with

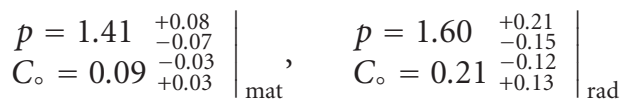

for the matter and radiation era, respectively. The loop number density distribution, for the matter era run, has been plotted in Figure 9. As Figure 8 already shows, the loop distribution takes more time to reach the scaling regime for the small loops. The relaxation bump is all the more so high and long than $\alpha$ is small. In the loop number density distribution, this effect appears as a minimal time decreasing value $\alpha_{\min }(\eta)$ such that the loop distribution is in scaling at $\alpha>\alpha_{\min }$. The redshift range probes by a FLRW string simulation is typically $\Delta z \simeq 10^{2}$, while for strings formed at the GUT energy scales, one expects a $\Delta z \simeq 10^{18}$ at nucleosynthesis. It is clear that, in the cosmological context, the string network has quite a time to relax; on all of the relevant observable length scales the loop distribution should be in scaling, that is, $\alpha_{\text {min }} \ll 1$. Since a power law distribution is scale-free, one concludes that a cosmologically stable string network does not exhibit loops of a particular size; this is not surprising since the only length scale involved is the distance to the horizon. These numerical results can be analytically recovered in the framework of the Polchinski-Rocha model [179]. The expected loop number density distribution have been explicitly derived by Rocha in [182] with a predicted power $p=1.5$ for the matter era and $p=1.8$ in the radiation era. If not due to statistical errors, these small differences may be explained by the existence of additional fractal microstructure along the strings not considered in the analytical approach (see also Section 4). Of course, the previous statements hold provided the other physical effects which are not included in the simulation do not enter the game, as gravitational radiation and gravitational backreaction. The typical length scales at which they should play a role is typically a multiple of $\mathrm{GU}$, or some positive power of it [183, 184]. As shown in [182], gravitational radiation indeed cures the energy density divergence that one can extrapolate from Figure 9 when $\alpha \rightarrow 0$. Let us finally notice that although the long strings are defined by $\alpha>1$, there is also a small population of Kibble loops. Their typical size being the horizon-sized correlation length of the long string network, they can be defined to be those having $\alpha_{\infty}<\alpha<1$, where

$$
\alpha_{\infty}=\frac{1}{d_{\mathrm{h}}}\left(\frac{U}{\rho_{\infty}}\right)^{1 / 2}
$$




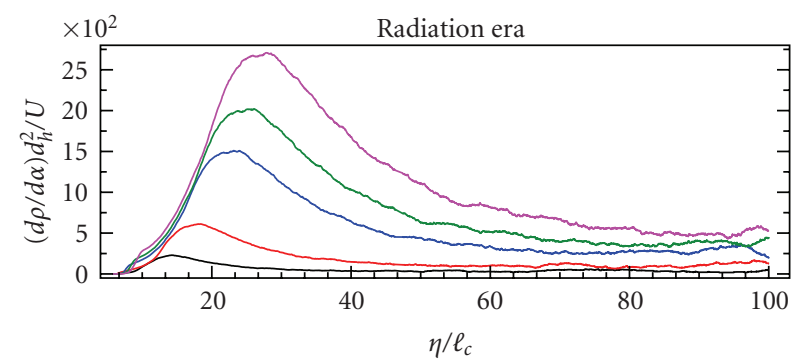

$$
\begin{aligned}
-\alpha & =0.035 \\
-\alpha & =0.02 \\
-\alpha & =0.012
\end{aligned}
$$

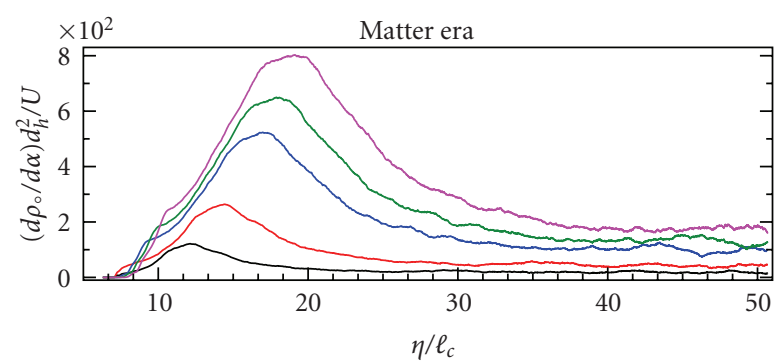

$\begin{aligned}-\alpha & =0.023 \\ -\alpha & =0.013 \\ -\alpha & =0.007\end{aligned}$

$$
\begin{aligned}
\alpha & =0.006 \\
\alpha & =0.005
\end{aligned}
$$

(a)

FIGURE 8: Evolution of the energy density distribution of $\alpha d_{\mathrm{h}}$-sized loops as a function of the conformal time in the radiation and matter era. After an overproduction regime, the energy density distribution scales as $1 / d_{h}^{2}$, as the long strings.

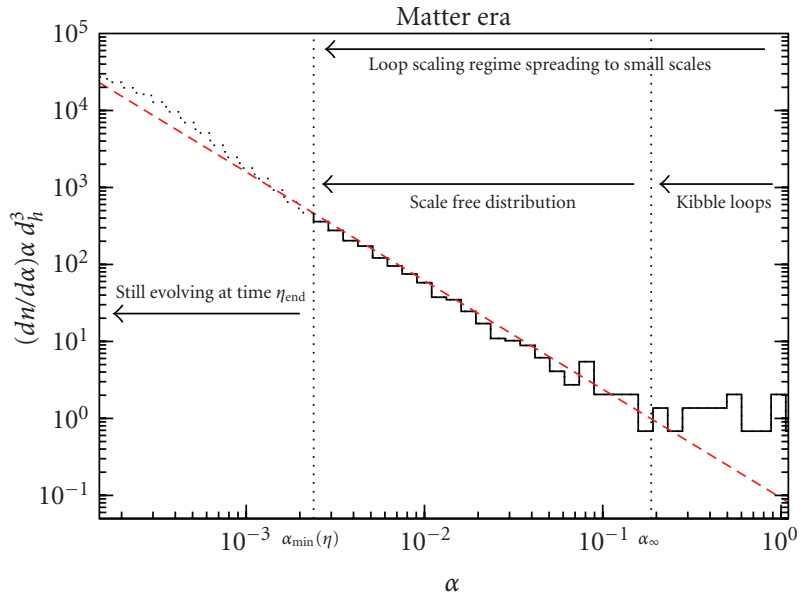

Figure 9: Loop number density distribution at the end of a $\left(100 \ell_{c}\right)^{3}$ matter era numerical simulation. Apart for a few Kibble loops $\left(\alpha_{\infty}<\right.$ $\alpha<1$ ), all loops of size $\ell=\alpha d_{h}$ with $\alpha>\alpha_{\text {min }}$ follow a scaling regime in which their number density is a power law. Smaller loops are still in the relaxation regime and will enter their scaling regime later (see Figure 10). The red dashed line if the best power law fit of the scaling function $\delta(\alpha)$ is given by (44).

3.5. Relaxation Towards Scaling: Memory of The Initial Conditions. Although of less-cosmological interest, the relaxation of the loop energy density distribution towards its scaling regime shows interesting properties which could explain some of the differences observed between Abelian Higgs field simulation and NG simulations. In the left panel of Figure 10, we have plotted the loop energy density distribution for loops smaller than the ones in scaling. At the end of the numerical simulation, these length scales are those having $\alpha<\alpha_{\min }$. This plot shows that the formation of the smallest loops is a delayed mechanism which suggests that a cascading process takes place from the initial string network configuration. For $\alpha \simeq 10^{-5}$, only the increasing part of the relaxation bump appears at the end of the simulation whereas the decaying towards scaling is still visible for the larger $\alpha$. On the right panel, we have plotted the loop number density distribution at the end of the simulation, that is, at the time $\eta / \ell_{c}=$ 50 in the left panel. The loop number density distribution deviates from the asymptotic scaling distribution on two typical length scales.

Firstly, an overdensity compared to scaling is situated at $\alpha_{c}=\ell_{c} / d_{h} \propto 1 / t$. In other words, there is an overproduction of loops with a typical size equal to the initial correlation length of the string network. Although one expects the system to retain some memory of the initial conditions during the relaxation, it may appear surprising that, in spite of the expansion of the universe, the physical length scale of these loops remains the same. A physical interpretation is that $\ell_{c} \ll d_{h}$ which suggests that, at those small length scales, the system decouples from the Hubble flow. More quantitatively, this effect can be explained in the context of the three scale models [176]. Under some assumptions, the string small-scale correlations can indeed sustain a constant physical length.

Then, there is the overall peak of the loop number density distribution at $\alpha_{r}=\ell_{r} / d_{h} \propto 1 / t$. Most of the loops which are not in scaling have this size at the end of the simulation. In fact, one can check that these loops start appearing soon after the beginning of the string evolution. This length scale is, again, at a constant physical length $\ell_{r}$ and is associated with a purely numerical effect [146]. As discussed, a numerical string is discretised with $N_{\text {ppcl }}$ points. The Bennett-Bouchet code at the basis of the simulations presented here uses an adaptive griding algorithm meaning that loops of any physical size can be formed [144]. The only restriction is that, at a given time, a loop is an object of at least three points. Consequently, when the initial string network starts its evolution, loops smaller than $\ell_{r}=\ell_{c} \times 3 / N_{\text {ppcl }}$ cannot be formed. The existence of a finite numerical resolution therefore adds some unwanted initial correlations of length $\ell_{r}$. Notice that this is not a cutoff but indeed an extracorrelation.

As a basic consequence, one should not trust an NG simulation at those length scales. However, the fact that the initial string network violently relaxes towards scaling by emitting loops at the smallest available correlation lengths has still some physical significance [185]. What happens if we 


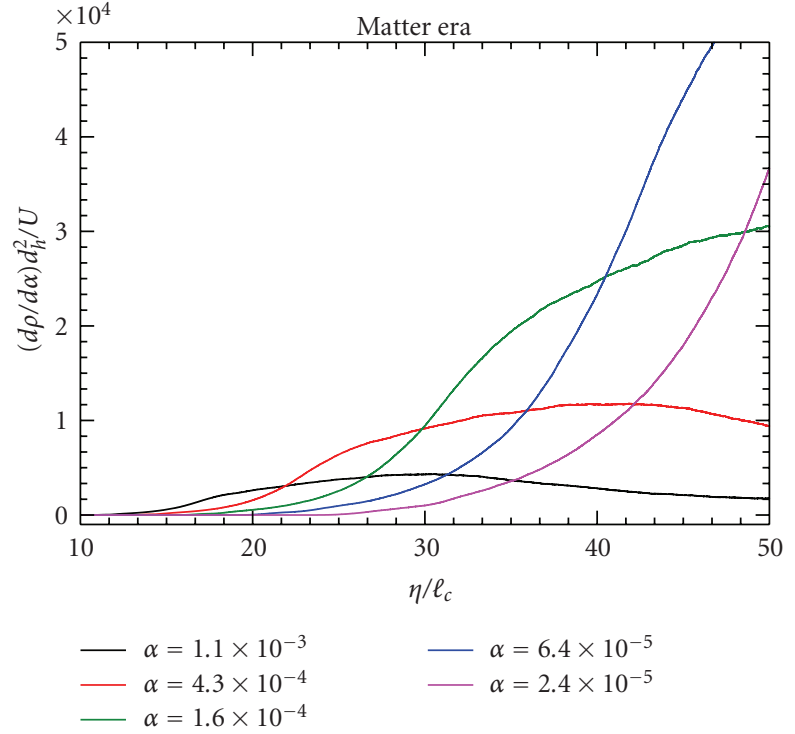

(a)

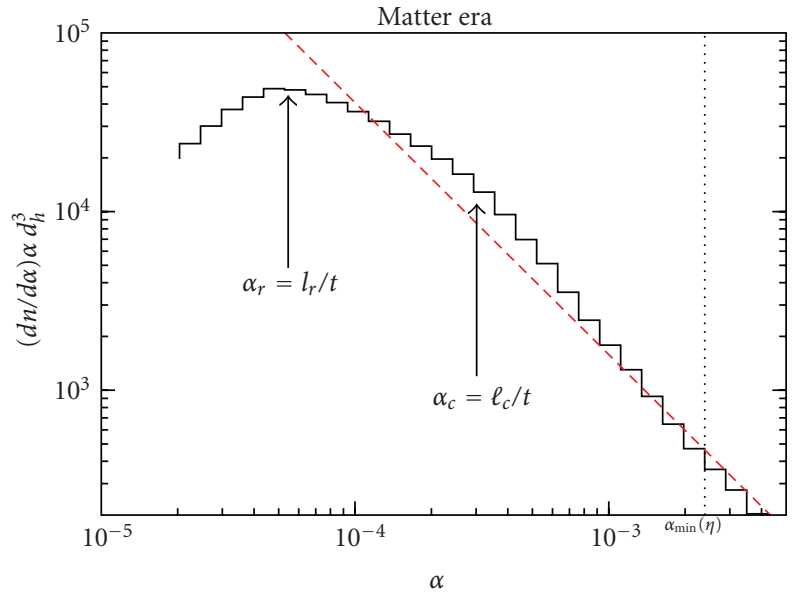

(b)

FIGURE 10: Loop energy density distribution with respect of the conformal time for the smallest loops. By the end of the numerical simulation, these loops have not yet reached their scaling behaviour (a). (b) is the corresponding loop number density distribution at the end of the simulation $\left(\eta / \ell_{c}=50\right)$. Compared to the asymptotic scaling power law (red dashed), the loops in the relaxation regime are preferentially produced at fixed physical lengths given by the initial correlations present in the VV network.

increase $N_{\text {ppcl }} \rightarrow \infty$ ? As discussed in [146], the larger length scales are not affected and only the overall peak is shifted around the new $\ell_{r} \rightarrow 0$. At these length scales, it is clear that using an NG string to describe a network of topological defects would break down and a reasonable assumption is to assume that the network will now relax by losing energy through the relevant physical mechanism available at those $\ell_{r} \rightarrow 0$ distances. In Abelian Higgs simulation, most of the network energy is emitted through field radiation, up to the point that almost no loop are observed in the simulations $[75,77]$. Abelian simulation suffers from low resolution compared to NG ones and this has been a subject of debate to decide whether or not this could explain the absence of loops $[76,77]$. The above NG results clearly support that particle and/or gravitational waves; emission is an important mechanism which certainly dominate the relaxation regime. However, when the scaling regime progressively takes place, from large to small length scales, the loop formation mechanism should become dominant. One may speculate that it is not clearly observed in Abelian simulations due to its delayed appearance, as it is actually the case in NG simulations when we compare it to the formation of $\ell_{r}$-sized loops. (A hint for this is the compatibility of the Abelian Higgs string correlators with the Polchinski-Rocha model [77]; this one explaining the NG loop distribution [182].)

\section{Cosmic Microwave Background Anisotropies and Non-Gaussianities}

At this point, numerical simulations of cosmic string evolution give us the means to derive observable predictions. As should be clear from the previous section, some structures in the numerical simulations are not supposed to be present after a realistic expansion factor of $10^{18}$; these are the structures still in the relaxation regime and such that $\alpha<$ $\alpha_{\min }$, at any simulation time. In the following, we will denote by "systematic errors", the uncertainties inherent to the presence of such nonscaling structures when deriving observable predictions from NG numerical simulations.

4.1. Unequal Time Correlators. The first method used to derive CMB anisotropies has been introduced in [186] and applied in [79, 186-191] for global topological defects and recently in $[192,193]$ for the Abelian strings.

Cosmic strings are active sources of gravitational perturbations [41, 195] which means that the equations of motion of their induced linear perturbation is of the form

$$
\mathscr{D} \mathcal{P}=\&,
$$

where $\mathscr{D}$ is a time differential operator, $\mathcal{P}$ the perturbation in the energy density, or velocity, and so forth, which is directly related to the $\mathrm{CMB}$ temperature anisotropies. Here $\&$ denotes the source terms, that is, the string stress tensor. From the Green's function $g$ of this equation, one gets, today (at $\eta_{0}$ ) and in Fourier space

$$
\mathcal{P}\left(k, \eta_{0}\right) \propto \int g_{k}(\eta) \delta(\eta, k) \mathrm{d} \eta .
$$

The two-point correlator reads

$$
\begin{aligned}
& \left\langle\mathcal{P}^{\dagger}\left(\eta_{0}, k\right) \mathcal{P}\left(\eta_{0}, k\right)\right\rangle \\
& \propto \iint^{\eta_{0}} g_{k}^{\dagger}\left(\eta^{\prime}\right) g_{k}(\eta)\left\langle\delta^{\dagger}\left(\eta^{\prime}, k\right) \delta(\eta, k)\right\rangle \mathrm{d} \eta \mathrm{d} \eta^{\prime},
\end{aligned}
$$




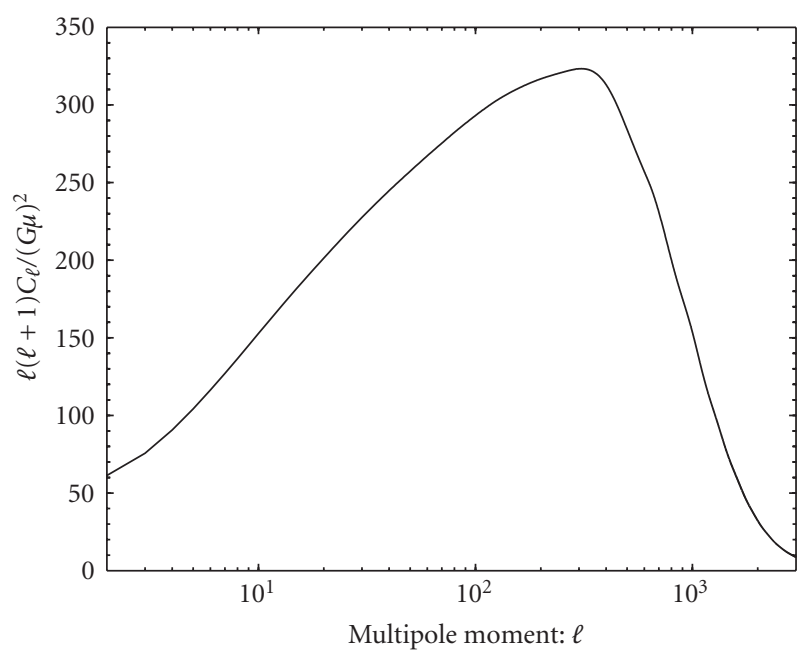

Figure 11: CMB temperature power spectrum induced by a network of Abelian cosmic strings and derived from the unequal time correlators method. Figure extracted from [194].

and its determination requires a full-time knowledge of the source term $s(\eta, k)$ for each mode. Since it is impossible to carry out a simulation over the whole cosmological history, the scaling properties of the cosmic string network can be used to analytically extrapolate the source terms over the required ranges. As shown in [189], as long as the cosmic string network is in a scaling regime, the source terms are the stress tensor components and assume the form

$$
\left\langle T_{\mu \nu}(k, \eta) T_{\rho \sigma}^{*}\left(k, \eta^{\prime}\right)\right\rangle \propto \frac{1}{\sqrt{\eta \eta^{\prime}}} f_{\mu \nu \rho \sigma}\left(k \sqrt{\eta \eta^{\prime}}, \frac{\eta}{\eta^{\prime}}\right) .
$$

Numerical simulations are actually used to determine the scaling functions $f_{\mu \nu \rho \sigma}$. In Figure 11, we have represented the temperature anisotropies derived in [194] using such a method from Abelian Higgs string simulations. The current $\mathrm{CMB}$ constraint on $G U$ comes from this power spectrum: at two-sigma, $G U<7 \times 10^{-7}[196]$.

4.2. Simulated Small Angle Maps. The previous constraint typically corresponds to a string contribution which cannot exceed $10 \%$ at the multipole moment $\ell=10$. On current observable angular scales, cosmic strings may only be a subdominant fraction of the overall $\mathrm{CMB}$ anisotropies. However, string-induced perturbations being non-Gaussian, as opposed to inflationary perturbations of quantum origin, one can go further than deriving the two-point function. Notice that, in principle, the unequal time correlator approach could be used to extrapolate the three- and higher $n$-point function by using the scaling properties of the string network. Another approach is to produce simulated maps of string-induced $\mathrm{CMB}$ anisotropies. Again, we face the problem of the small redshift range probed by the numerical simulations. By putting an observer inside the numerical simulation, such maps can only include stringy effects up to a finite redshift, typically $z \simeq 10^{2}$. The CMB anisotropies computed in this way are therefore only accurate on large

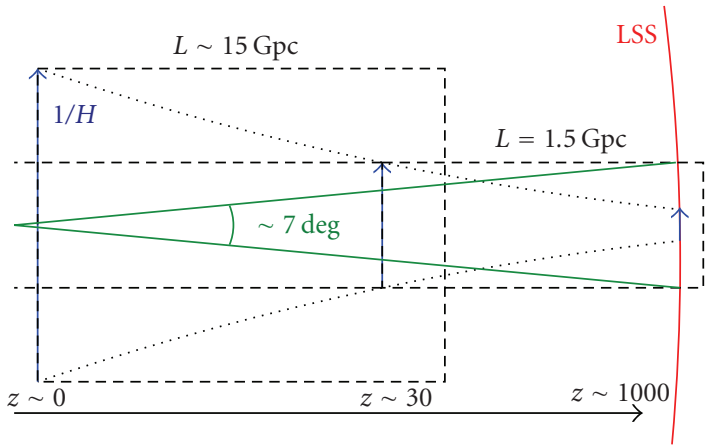

FIGURE 12: Stacking of two numerical simulations to compute the small angles CMB anisotropies induced by NG strings [197]. The dashed rectangles represent the redshift and angular extension of two numerical simulations used to evaluate (52). The first starts at last scattering and stops at $z \simeq 30$ while the second starts at $z \simeq$ 30 and ends now, up to a small overlapping to ensure relaxation towards scaling.

angular scales but can produce full sky maps [198-201]. This limitation can be avoided by stacking maps from different redshifts, an approach outlined in [37] and applied in [197, 202].

Simulations with the observer outside of the numerical box are not well suited for a full-sky map reconstruction, but are perfectly designed for the small angular scales. The reason being that cosmic strings are incessantly sourcing the $\mathrm{CMB}$ fluctuations since the last scattering surface, and contrary to the perturbations of inflationary origin, this part cannot be affected by Silk damping. Therefore, at small angular scales, one expects the strings' signature in the $\mathrm{CMB}$ temperature fluctuations to be dominated by their integrated Sachs-Wolfe (ISW) effect from the last scattering surface [202]. In the temporal gauge $\left(\tau=X^{0}=\eta\right)$, the NG stress tensor derived from (26) reads

$$
T^{\mu \nu}=\frac{U}{\sqrt{-g}} \int \mathrm{d} \sigma\left(\varepsilon \dot{X}^{\mu} \dot{X}^{\nu}-\frac{1}{\varepsilon} X^{\prime} X^{\prime}\right) \delta^{3}(\vec{x}-\vec{X}) .
$$

In the flat sky approximation, well suited for angles typically smaller than the Hubble angular size at the epoch of interest, Hindmarsh has shown that the ISW temperature anisotropies induced by NG strings can be simplified to $[203,204]$

$$
\widehat{\Theta}_{\mathbf{k}} \simeq \frac{8 \pi \mathrm{i} G U}{\mathbf{k}^{2}} \int_{\vec{X} \cap \vec{x}_{y}}(\mathbf{u} \cdot \mathbf{k}) \mathrm{e}^{-\mathrm{i} \mathbf{k} \cdot \mathbf{X}} \varepsilon \mathrm{d} \sigma,
$$

where $\Theta(\mathbf{x}) \equiv \delta T(\mathbf{x}) / T_{\text {CMB }}$. The wave vector $\mathbf{k}$ denotes the transverse component of the three-dimensional vector $\vec{k}$ with respect to the line of sight $\hat{n}$, whereas, in the temporal gauge, $\vec{u}$ encodes the string stress tensor distortions of the photon temperature and reads

$$
\vec{u}=\dot{\vec{X}}-\frac{(\hat{n} \cdot \dot{\vec{X}}) \cdot \dot{\vec{X}}}{1+\hat{n} \cdot \dot{\vec{X}}} .
$$


As can be seen in (51), only the strings that intercept the photon path $\vec{x}_{\gamma}$ can imprint their signature in the CMB temperature fluctuations. The previous expression is nothing but the Gott-Kaiser-Stebbins effect in the temporal gauge $[54,203,205]$. As a result, the knowledge of $\mathbf{u}$, and therefore of the string trajectories $\mathbf{X}$, is only required on our past light cone. In the context of string numerical simulations, the trajectories of all strings are computed during all of the numerical simulation time. Therefore, to compute $\mathbf{u}$, one only needs to determine which parts of the string network intercept our past light cone and at what time.

In order to be able to generate a significant amount of maps using such a method, it is more convenient to stack two medium sized $\left(50 \ell_{c}\right)^{3}$ simulations along the lines sketched in Figure 12. The first one starts at the last scattering surface and ends at a redshift fixed by the maximum expansion factor achievable in the numerical box. For the simulations we performed, initially $d_{h} \simeq 0.185$ (in unit of the fixed comoving simulation volume), which corresponds to $1.7 \mathrm{Gpc}$ and a field of view of $\theta_{\text {fov }} \simeq 7.2^{\circ}$ (for the current fiducial cosmological parameters [44]). Such a run ends after a 30 -fold increase in expansion factor, corresponding to a redshift $z \simeq 36$. We then propagate the photons perturbed by the first run into a second numerical simulation of the same size but starting at $z_{\mathrm{i}} \simeq 36$. For another 30fold increase in expansion factor, this run ends at $z \simeq$ 0.3 . As can be seen in Figure 12, the second simulation represents a much larger real volume than the first one and therefore subtends a greater angle in the sky. As a result, only the subpart of the second run that matches the angle subtended by the first simulation is actually used. As we will see later on, the CMB temperature maps are weakly sensitive to the string network at low redshifts, simply because there are almost no strings intercepting our past light cone in a recent past, which makes this technique perfectly acceptable. In practice, each of these numerical simulations is started before the redshifts mentioned, in order to give the cosmic string network enough time to relax toward its stable cosmological configuration. As discussed in the previous section, one has to make sure that the structures (strings and loops) we are interested in have indeed reached their scaling behavior during the numerical runs. We switch on the photon propagation inside the runs only after making sure all the large structures (infinite strings and loops) are in their scaling regime. This can be checked by monitoring the evolution of the energy density distributions, and we have chosen to start the photons' propagation when all loops larger than a third of the horizon size are in scaling. This cutoff is then dynamically pushed to smaller values to include all the loops entering the scaling regime at later times. The cutoff time dependence is simply the function $\alpha_{\min }(\eta)$ and can be deduced from the loop distribution relaxation times derived in Section 3.4.2. The resulting CMB temperature map is displayed in the left panel of Figure 13 whereas the right panel shows the string paths projected onto our past light cone. Again, these maps are only representative at small angular scales. On larger angles, they represent only the ISW contribution to the overall string anisotropies; for instance, Doppler contributions coming from photon decoupling at last scattering are dominant around $\ell=300-400$ (see Figure 11).

The discussion on systematic effects coming from the presence of loops not yet in scaling can be found in [46]. In fact, they have only a small effect. The physical reason being that, due to scaling, the long strings are still the main source of CMB anisotropies even at (reasonably) small angles. Indeed, there are always roughly ten strings per Hubble volume at each time, which means that a patch of $0.8^{\circ}$ is at least crossed by the ten long strings being there at last scattering, plus a few others from lower redshifts.

4.3. Skewness and Kurtosis. The most basic statistical test that can be performed from a set of small angle CMB maps is to plot the one-point function of the temperature anisotropies. As can be seen in Figure 14, the temperature anisotropies induced by cosmic strings are clearly non-Gaussian. From a set of 300 independent CMB maps, one finds the mean sample skewness to be negative

$$
g_{1} \equiv\left\langle\overline{(\Theta-\bar{\Theta})^{3}} \frac{\overline{\left(\sigma^{3}\right.}}{\sigma^{3}} \simeq-0.22 \pm 0.12,\right.
$$

where the brackets stand for the mean over different realisations while the bar denotes averaging on each map. The variance itself averages to

$$
\sigma^{2} \equiv\left\langle\overline{(\Theta-\bar{\Theta})^{2}}\right\rangle \simeq(150.7 \pm 18)(G U)^{2} .
$$

The quoted errors are statistical and refer to the square root of the variance between the different realisations. Similarly, the mean kurtosis averages to

$$
g_{2} \equiv\left\langle\overline{\left(\overline{(\Theta-\bar{\Theta})^{4}}\right.} \frac{\overline{\sigma^{4}}}{}\right\rangle-3 \simeq 0.69 \pm 0.29
$$

An analytical approach extending these results to cosmic superstrings can be found in [206]. A simple way to look for strings is to search for large (but rare) temperature fluctuations. Deviations from Gaussianity start to be significant, let us say by a factor of two, only in the tails when the probability distribution becomes typically lower than $10^{-6}$.

4.4. Real Space Methods. Strings induce step-like discontinuities in the CMB anisotropies and various methods have been designed to probe the non-Gaussianities associated with them.

Multifractal analysis [207] has the advantage of being directly applicable to the time-ordered data retrieved when a CMB telescope scans the sky. As opposed to the fractal dimension of a set which measures how sparse it is, the multifractal spectrum of a measure defined over a set gives how many and which fractal dimensions there are. In the context of cosmic strings, this method has been applied in [208] on one dimensional scan of maps similar to the one in Figure 13, the measure being defined by

$$
\mu(i)=[\Theta(i)-\Theta(i+1)]^{2},
$$




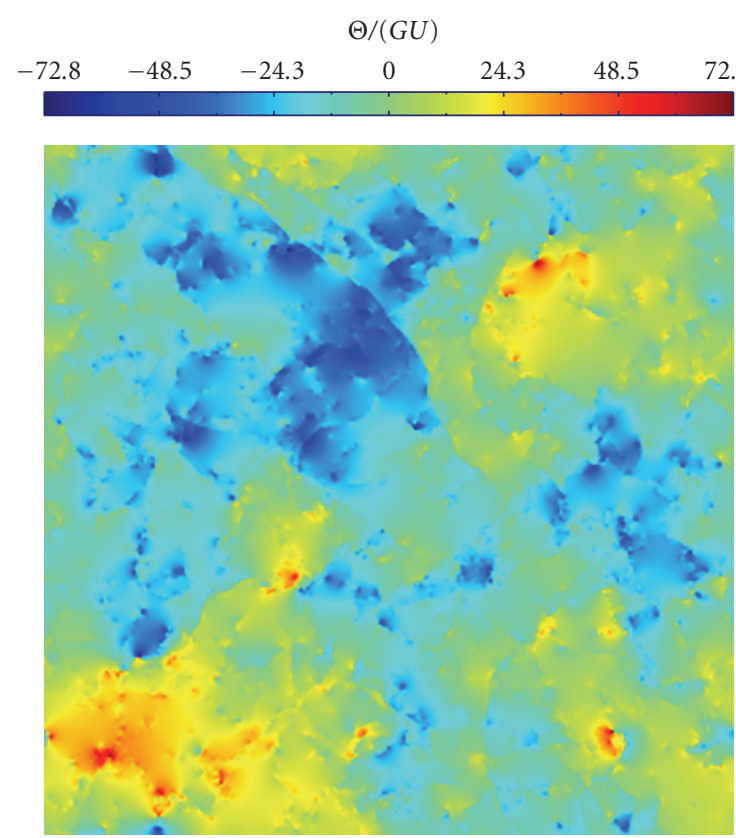

(a)

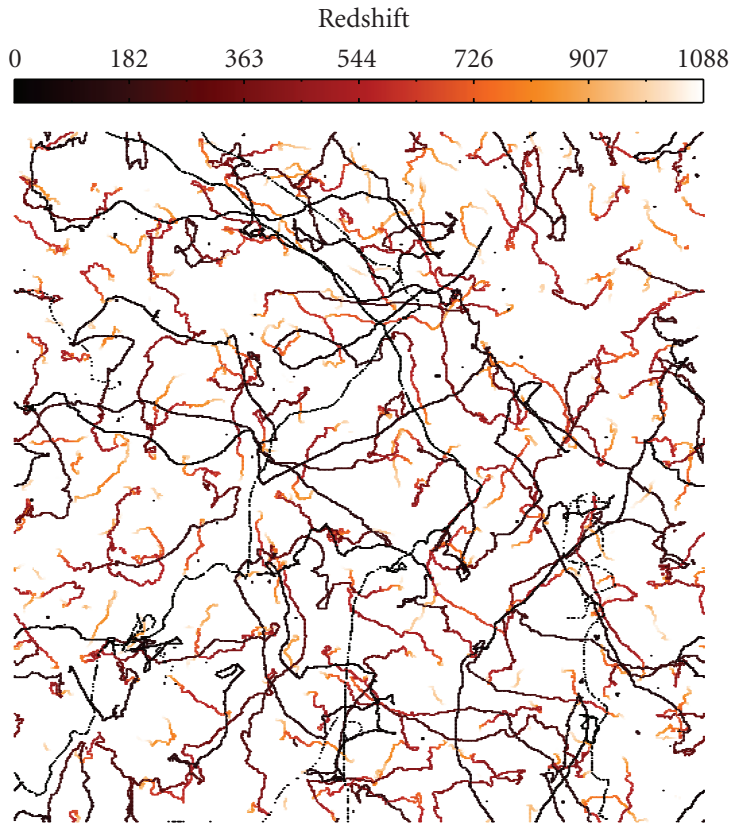

(b)

Figure 13: String-induced CMB temperature fluctuations on a $7.2^{\circ}$ field of view [46]. Because of their cosmological scaling, most of the long strings intercept our past light cone close to the last scattering surface. As can be seen in the right image, the edges in the temperature patterns can be identified to strings intercepting our past light cone. Note that active regions corresponding to string intersection and loop formation events lead to the bright spots in these maps.

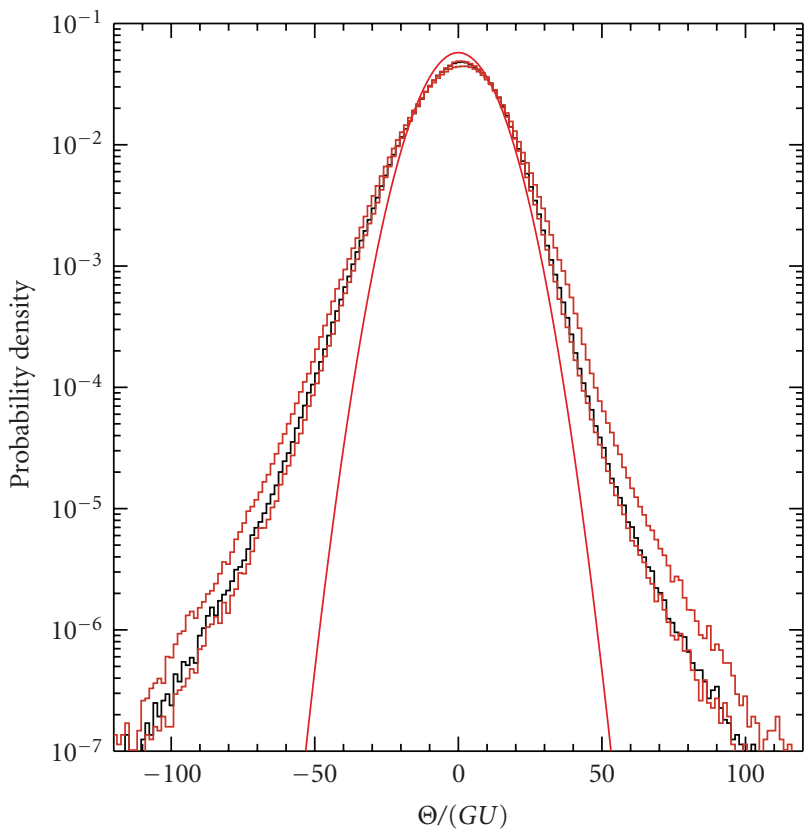

FIGURE 14: The probability distribution function of CMB temperature fluctuations induced by NG cosmic strings. The orange curves quantify systematic errors coming from the string simulations by including nonscaling loops, or by removing all loops. Deviations from Gaussianity are clearly apparent in the tails of the distribution, as well as from the negative skewness. where the integer $i$ labels a point along the scan. The multifractal properties of this measure have been shown to be distinctive enough to detect strings, compared to a Gaussian signal, but only when the detector resolution is sufficiently good. One may wonder, under multifractality, how a non-Gaussian string pattern could be distinguished from other non-Gaussian sources. In fact, (56) consists in taking the gradient of the induced CMB fluctuations along the scan. Step-like discontinuities, passed over a gradient filter, become one-dimensional delta functions, and this is a definite string feature that can only be altered by the beam experiment. Denoting by $\alpha$ and $\beta$ the horizontal and vertical angular coordinates, the gradient magnitude $|\nabla \Theta|$ of the temperature anisotropies is defined by

$$
|\nabla \Theta| \equiv \sqrt{\left(\frac{\mathrm{d} \Theta}{\mathrm{d} \alpha}\right)^{2}+\left(\frac{\mathrm{d} \Theta}{\mathrm{d} \beta}\right)^{2}} .
$$

This definition makes it clear that for a finite temperature step, let us say $\Theta(\alpha, \beta)=\Theta_{0} \mathrm{H}\left(\alpha-\alpha_{0}\right)$, H being the Heaviside function, the resulting gradient magnitude is a Dirac distribution at the string location. In Figure 15, we have plotted the gradient magnitude of the temperature maps of Figure 13 as well as its convolved version with a Gaussian beam typical of the Planck satellite at $217 \mathrm{GHz}$. With a finite resolution beam, the discontinuities are now smoothed. Real space methods applied to string are therefore strongly sensitive to the angular resolution. Let us mention 


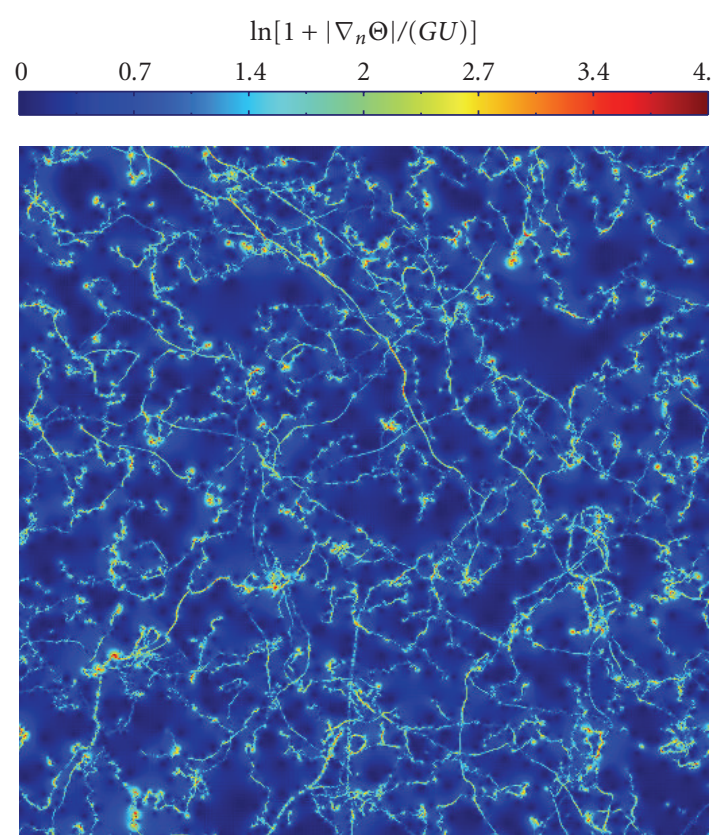

(a)

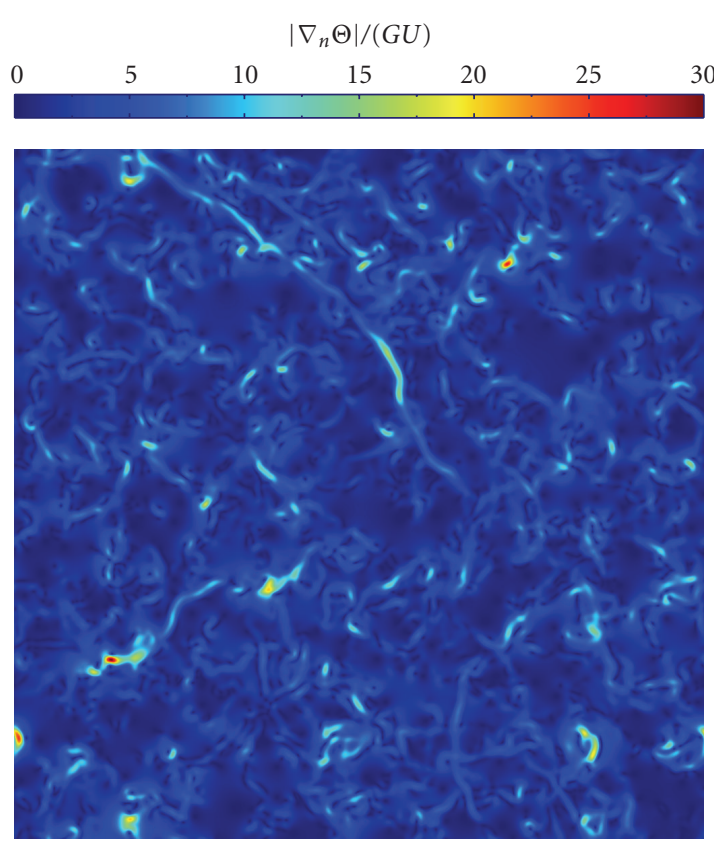

(b)

FIGURE 15: Normalized gradient magnitude of the string-induced temperature anisotropies shown in Figure 13(a). A logarithmic scale has been used to enhance the contrast by preventing the bright spots from saturating the color scale. The right panel is the gradient magnitude obtained after convolution by a Planck-like Gaussian beam of resolution $5^{\prime}$. Notice that the color scale is back to linear, most of the bright spots being now smoothed by the beam.

that wavelet analysis methods have been also explored in this context $[209,210]$ or to produce cleaner maps [211].

Directional gradients, obtained by variations with respect to either $\alpha$ or $\beta$, have been discussed in [205] in the context of Minkowski functionals. They are again found to provide a more distinctive non-Gaussian behaviour than the original temperature map for the contour length and genus.

4.5. Temperature Power Spectrum. Moving to Fourier space, the small angle $\mathrm{CMB}$ maps also permit a determination of the power spectrum at large multipoles. In Figure 16, we have plotted its mean value over the different maps as well as the one-sigma statistical error around the mean. The overall power at $\ell=1000$ is [46]

$$
\left.\frac{\ell(\ell+1) C_{\ell}}{2 \pi}\right|_{\ell=1000} \simeq 14(G U)^{2},
$$

which is close to the value obtained in Abelian Higgs field simulation (see Figure 11). This is not so surprising since the long strings in both NG and Abelian Higgs simulation have a similar scaling evolution, and as explained above, long strings are the main sources of $\mathrm{CMB}$ anisotropies even at the small angles. The power law tail in Figure 16 is the direct consequence of the presence of strings at all times since the last scattering surface: one finds for $\ell \gg 1$ [46]

$$
\ell(\ell+1) C_{\ell} \propto \ell^{-p}, \quad \text { with } p=0.889_{-0.090}^{+0.001} \text {, }
$$

where only the systematic errors have been reported. Such a power law shows that cosmic strings have to become the

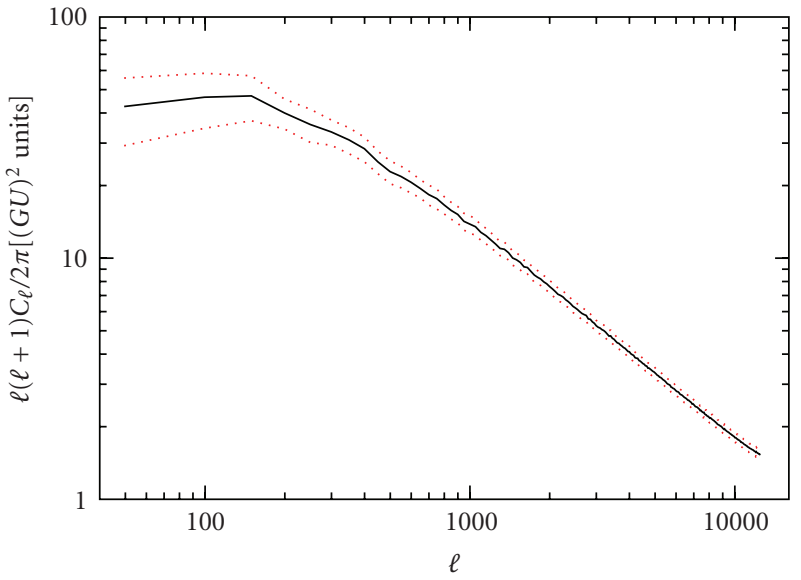

FIGURE 16: Mean angular power spectrum of the string-induced $\mathrm{CMB}$ anisotropies at small angular scales and its one sigma statistical errors (averaged over 300 maps). At "small" multipoles $\ell<500$, Doppler contributions from the last scattering surface are expected to be significant and this plot gives only the ISW component [46].

dominant primary source of $\mathrm{CMB}$ anisotropies at the small angular scales, the fluctuations of inflationary origin being killed by Silk damping at those multipoles. In Figure 17, we have plotted the respective contributions of strings and adiabatic anisotropies of inflationary origin. The cosmological parameters have been set to their fiducial values in the Lambda-Cold-Dark-Matter (LCDM) model and the string 


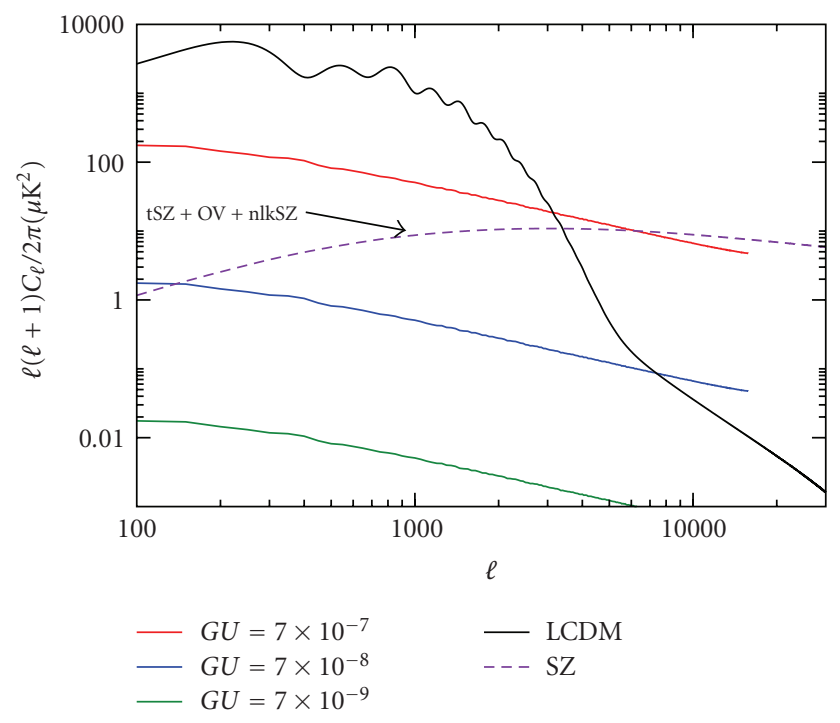

Figure 17: CMB temperature anisotropies from various sources, compared to the expected string contribution. Due to the Silk damping of primordial perturbations, string-induced anisotropies always become dominant for the large multipoles. However, an unresolved SZ effect may compromise such a clean signature [46].

energy density $U$ is compatible with the current bounds. For the current upper limit on $G U=7 \times 10^{-7}$, CMB anisotropies should become dominated by cosmic strings at $\ell \gtrsim 3000$. An unresolved Sunyaev-Zel'dovich (SZ) component may, however, compromise such a signal. Nevertheless, string induced anisotropies do not depend on the signal frequency whereas the SZ does, and one may hope to disentangle both [46].

4.6. Hindmarsh Approximation. As shown by Hindmarsh in [203], the power law behaviour of the NG string power spectrum at small angles can be analytically recovered. In Fourier space, the power spectrum of the string-induced $\mathrm{CMB}$ anisotropies $\Theta$ is defined by

$$
\left\langle\widehat{\Theta}_{\mathbf{k}_{1}} \widehat{\Theta}_{\mathbf{k}_{2}}\right\rangle=P\left(k_{1}\right)(2 \pi)^{2} \delta\left(\mathbf{k}_{1}+\mathbf{k}_{2}\right),
$$

the expression of $\widehat{\Theta}_{\mathbf{k}}$ being given by (51). Remembering that the string positions and velocity vectors have to be evaluated on the past light cone, it is more convenient to use the socalled light cone gauge. Instead of identifying the timelike worldsheet coordinate $\tau$ with the background time at the string event, one chooses instead to identify $\tau=X^{+} \equiv$ $X^{0}+X^{3}$. In this gauge, (51) simplifies to

$$
-k^{2} \widehat{\Theta}_{\mathbf{k}}=i \epsilon k_{A} \int \mathrm{d} \sigma \dot{X}^{A}(\sigma) e^{i \mathbf{k} \cdot \mathbf{X}(\sigma)},
$$

where we have defined

$$
\epsilon=8 \pi G U
$$

and where the capital indices are two-dimensional. The time parameter $\tau$ then labels the intersections of a set of null hyperplanes with the worldsheet. For our problem, all quantities have to be evaluated at $\tau=x^{+}=\eta+z$. In a field of view of formal area $\mathcal{A}=(2 \pi)^{2} \delta(0)$, one can express the power spectrum as

$$
P(k)=\epsilon^{2} \frac{k_{A} k_{B}}{A k^{4}} \int \mathrm{d} \sigma \mathrm{d} \sigma^{\prime}\left\langle\dot{X}^{A}(\sigma) \dot{X}^{B}\left(\sigma^{\prime}\right) e^{i \mathbf{k} \cdot\left[\mathbf{X}(\sigma)-\mathbf{X}\left(\sigma^{\prime}\right)\right]}\right\rangle .
$$

Adding the assumptions that both $\dot{X}^{A}$ and $X^{B}$ obey Gaussian statistics, all of the correlation functions of $\widehat{\Theta}_{k}$ can now be written in terms of two-point functions only. Using the same notation as in [203], the nonvanishing two-point functions are

$$
\begin{gathered}
\left\langle\dot{X}^{A}(\sigma) \dot{X}^{B}\left(\sigma^{\prime}\right)\right\rangle=\frac{1}{2} \delta^{A B} V\left(\sigma-\sigma^{\prime}\right), \\
\left\langle\dot{X}^{A}(\sigma) X^{B}\left(\sigma^{\prime}\right)\right\rangle=\frac{1}{2} \delta^{A B} M\left(\sigma-\sigma^{\prime}\right), \\
\left\langle X^{\prime}(\sigma) X^{B}\left(\sigma^{\prime}\right)\right\rangle=\frac{1}{2} \delta^{A B} T\left(\sigma-\sigma^{\prime}\right),
\end{gathered}
$$

as well as the quantities

$$
\begin{aligned}
\Gamma\left(\sigma-\sigma^{\prime}\right) & \equiv\left\langle\left[\mathbf{X}(\sigma)-\mathbf{X}\left(\sigma^{\prime}\right)\right]^{2}\right\rangle \\
& =\int_{\sigma^{\prime}}^{\sigma} \mathrm{d} \sigma_{1} \int_{\sigma^{\prime}}^{\sigma} \mathrm{d} \sigma_{2} T\left(\sigma_{1}-\sigma_{2}\right), \\
\Pi\left(\sigma-\sigma^{\prime}\right) & \equiv\left\langle\left[\mathbf{X}(\sigma)-\mathbf{X}\left(\sigma^{\prime}\right)\right] \cdot \dot{\mathbf{X}}\left(\sigma^{\prime}\right)\right\rangle \\
& =\int_{\sigma^{\prime}}^{\sigma} \mathrm{d} \sigma_{1} M\left(\sigma_{1}-\sigma^{\prime}\right) .
\end{aligned}
$$

The leading terms are given by $[203,212]$

$$
\begin{gathered}
V(\sigma) \longrightarrow\left\{\begin{array}{ll}
\bar{v}^{2}, & \sigma \longrightarrow 0 \\
0, & \sigma \longrightarrow \infty
\end{array}, \quad \Gamma(\sigma) \longrightarrow \begin{cases}\bar{t}^{2} \sigma^{2}, & \sigma \longrightarrow 0 \\
\hat{\xi} \sigma, & \sigma \longrightarrow \infty,\end{cases} \right. \\
\Pi(\sigma) \longrightarrow \begin{cases}\frac{1}{2} \frac{c_{0}}{\hat{\xi}} \sigma^{2}, & \sigma \longrightarrow 0, \\
0, & \sigma \longrightarrow \infty,\end{cases}
\end{gathered}
$$

where we have defined

$$
\hat{\xi}=\Gamma^{\prime}(\infty), \quad \bar{v}^{2}=\left\langle\dot{\mathbf{X}}^{2}\right\rangle, \quad \bar{t}^{2}=\left\langle\dot{X}^{2}\right\rangle, \quad c_{0}=\hat{\xi}\langle\dot{X} \cdot \dot{\mathbf{X}}\rangle .
$$

The correlation length $\hat{\xi}$ is the projected correlation length on the past light cone, $\bar{t}^{2}$ is the mean square projected tangent vector, $\bar{v}^{2}$ is the mean square projected velocity and $c_{0}$ the correlation between projected velocity and curvature. From these assumptions, (63) reduces to

$P(k)$

$$
=\frac{\epsilon^{2}}{2 \mathcal{A} k^{2}} \int \mathrm{d} \sigma \mathrm{d} \sigma^{\prime}\left[V\left(\sigma-\sigma^{\prime}\right)+\frac{1}{2} k^{2} \Pi^{2}\left(\sigma-\sigma^{\prime}\right)\right] e^{-k^{2} \Gamma\left(\sigma-\sigma^{\prime}\right) / 4} .
$$


When $k \hat{\xi}$ gets large, the terms involving the mixed correlator $M$ can be shown to be subdominant and only the first term remains

$$
P(k)=\frac{\epsilon^{2}}{4 \mathscr{A} k^{2}} \int \mathrm{d} \sigma_{+} \mathrm{d} \sigma_{-} V\left(\sigma_{-}\right) e^{-k^{2} \Gamma\left(\sigma_{-}\right) / 4},
$$

where $\sigma_{ \pm}=\sigma \pm \sigma^{\prime}$. Denoting by $L$ the total transverse lightcone gauge length of string in the box of area $\mathcal{A}$, one gets

$$
k^{2} P(k) \simeq \epsilon^{2} \sqrt{\pi} \frac{L \hat{\xi}}{\mathcal{A}} \frac{\bar{v}^{2}}{\bar{t}} \frac{1}{(k \hat{\xi})} .
$$

At small angles, the wave number $k^{2} \simeq \ell(\ell+1)$ and $(74)$ predicts that $\ell(\ell+1) C_{\ell} \propto \ell^{-1}$. The small difference with (59) is suggestive of a cloud of zero-dimensional objects along the string worldsheet which may be the signature of small loop production in the NG numerical simulations. Let us stress that (74) is not "primordial" but directly approximate the observed angular power spectrum of the CMB temperature anisotropies.

\subsection{Bispectrum}

4.7.1. Analytical Approach. The success of Hindmarsh approximation to describe the small angular CMB anisotropies power spectrum suggests it can be applied to higher $n$-point functions. In [212], this method was used to derive the bispectrum defined from the three points function by

$$
\left\langle\widehat{\Theta}_{\mathbf{k}_{1}} \widehat{\Theta}_{\mathbf{k}_{2}} \widehat{\Theta}_{\mathbf{k}_{3}}\right\rangle=B\left(\mathbf{k}_{1}, \mathbf{k}_{2}, \mathbf{k}_{3}\right)(2 \pi)^{2} \delta\left(\mathbf{k}_{1}+\mathbf{k}_{2}+\mathbf{k}_{3}\right) .
$$

Plugging (61) into the previous expression gives

$$
\begin{aligned}
& B\left(\mathbf{k}_{1}, \mathbf{k}_{2}, \mathbf{k}_{3}\right) \\
& =i \epsilon^{3} \frac{1}{\mathcal{A}} \frac{k_{1_{A}} k_{2_{B}} k_{3_{C}}}{k_{1}^{2} k_{2}^{2} k_{3}^{2}} \int \mathrm{d} \sigma_{1} \mathrm{~d} \sigma_{2} \mathrm{~d} \sigma_{3}\left\langle\dot{X}_{1}^{A} \dot{X}_{2}^{B} \dot{X}_{3}^{C} e^{i \delta^{a b} \mathbf{k}_{a} \cdot \mathbf{X}_{b}}\right\rangle,
\end{aligned}
$$

with $\dot{X}_{a}^{A}=\dot{X}^{A}\left(\sigma_{a}\right), a, b \in\{1,2,3\}$, and $\mathbf{k}_{1}+\mathbf{k}_{2}+\mathbf{k}_{3}=0$. With the Gaussian assumption, the ensemble average of the string observables is lengthy but straightforward and the final result reads [212]

$$
\begin{aligned}
& B\left(\mathbf{k}_{1}, \mathbf{k}_{2}, \mathbf{k}_{3}\right) \\
& =-\epsilon^{3} \pi c_{0} \frac{\bar{v}^{2}}{\bar{t}^{4}} \frac{L \hat{\xi}}{\mathcal{A}} \frac{1}{\hat{\xi}^{2}} \frac{1}{k_{1}^{2} k_{2}^{2} k_{3}^{2}}\left[\frac{k_{1}^{4} \kappa_{23}+k_{2}^{4} \kappa_{31}+k_{3}^{4} \kappa_{12}}{\left(\kappa_{23} \kappa_{31}+\kappa_{12} \kappa_{31}+\kappa_{12} \kappa_{23}\right)^{3 / 2}}\right] .
\end{aligned}
$$

The quantities $\kappa_{a b}$ are shorthand for the scalar products $\kappa_{a b} \equiv-\mathbf{k}_{a} \cdot \mathbf{k}_{b}$. In the same way as for the power spectrum, this expression directly gives the bispectrum of the CMB temperature anisotropies. Its overall dependence varies as $1 / k^{6}$. Its sign depends on the sign of $c_{0}$ defined in (71), and contrary to what one could naively expect $c_{0} \neq 0$ : the projected string velocity and curvature vectors are correlated. This can be shown by starting again from the equations of motion (29), but this time, in the light cone gauge. The equation of motion for $X^{+}$gives

$$
\frac{\dot{\varepsilon}}{\varepsilon}+2 \mathscr{H}\left(\dot{X}^{0}+\dot{X}^{2}\right)=0
$$

whereas the equation for the transverse components is

$$
\ddot{\mathbf{X}}+2 \mathscr{H} \frac{1}{\varepsilon^{2}}\left(\mathbf{X}^{\prime}\right) \dot{\mathbf{X}}-\frac{1}{\varepsilon} \frac{\partial}{\partial \sigma}\left(\frac{1}{\varepsilon} \frac{\partial \mathbf{X}}{\partial \sigma}\right)-2 \mathscr{H} \frac{1}{\varepsilon^{2}}(\dot{\mathbf{X}} \cdot \stackrel{\mathbf{X}}{ }) \stackrel{\prime}{\mathbf{X}}=0 .
$$

In a FLRW background, assuming that $\left\langle\dot{\mathbf{X}}^{2}\right\rangle$ is constant, and neglecting higher-order correlations between $\mathscr{H}, \dot{\mathbf{X}}$, and $\mathbf{X}$, we find

$$
\left\langle\frac{\partial^{2} \mathbf{X}}{\partial s^{2}} \cdot \dot{\mathbf{X}}\right\rangle=2 \overline{\mathscr{H}}\left\langle\left(\frac{\partial \mathbf{X}}{\partial s}\right)^{2} \dot{\mathbf{X}}^{2}\right\rangle-2 \overline{\mathscr{H}}\left\langle\left(\dot{\mathbf{X}} \cdot \frac{\partial \mathbf{X}}{\partial s}\right)^{2}\right\rangle,
$$

where we have defined $\mathrm{d} s=\varepsilon \mathrm{d} \sigma$, and where $\overline{\mathscr{H}}$ is the averaged conformal Hubble parameter. Still assuming that the ensemble is approximately Gaussian in $\dot{\mathbf{X}}$ and $\mathbf{X}^{\prime} / \varepsilon$, the right hand side reduces to

$$
\left\langle\frac{\partial^{2} \mathbf{X}}{\partial s^{2}} \cdot \dot{\mathbf{X}}\right\rangle=\overline{\mathscr{H}}\left(\left\langle\dot{\mathbf{X}}^{2}\right\rangle\left\langle\mathbf{X}^{2}\right\rangle-\langle\dot{\mathbf{X}} \cdot \dot{\mathbf{X}}\rangle^{2}\right)
$$

The last term vanishes and the cross correlator $\overline{\mathscr{H}} \bar{v}^{2} \bar{t}^{2}$ is positive; from (71), we deduce that $c_{0}>0$. It is interesting to notice that $c_{0}$ would vanish in Minkowski spacetime, which can be viewed as a consequence of time reversal invariance. The existence of a cosmic string bispectrum is the consequence of the breaking of the time reversal invariance in a FLRW background.

An illustrative example is to apply (77) to the isosceles triangle configurations in Fourier space such that

$$
\left|\mathbf{k}_{1}\right|=\left|\mathbf{k}_{2}\right|=k, \quad\left|\mathbf{k}_{3}\right|=2 k \sin \frac{\theta}{2}
$$

where $\theta$ denotes the angle between the wavevectors $\mathbf{k}_{1}$ and $\mathbf{k}_{2}$. The isosceles bispectrum reads

$$
B_{\ell \ell \theta}(k, \theta)=-\epsilon^{3} \pi c_{0} \frac{\bar{v}^{2}}{\bar{t}^{4}} \frac{L \hat{\xi}}{\mathcal{A}} \frac{1}{\hat{\xi}^{2} k^{6}} \frac{1+4 \cos \theta \sin ^{2}(\theta / 2)}{\sin ^{3} \theta} .
$$

Notice that for $\theta=\pi / 3$, we obtain the peculiar case of an equilateral triangle. In Figure 18, we have plotted the angle dependency of the isosceles bispectrum. These configurations are amplified as $1 / \theta^{3}$ in the two flat triangle limits for which either $\theta \rightarrow 0$ or $\theta \rightarrow \pi$. Both of these configurations are therefore better suited than the equilateral one to characterize the strings. As suggested by the real space searches, the strings can produce a strong bispectrum signal only if the detector resolution is sufficiently good. Assuming a beam resolution of $5^{\prime}$ means that the $7.2^{\circ}$ field of view would contain at maximum roughly $80^{2}$ Fourier modes. Consequently, the smallest values of $\theta$ achievable would be around $\theta>0.03$ radians, with only a few modes saturating this bound. 


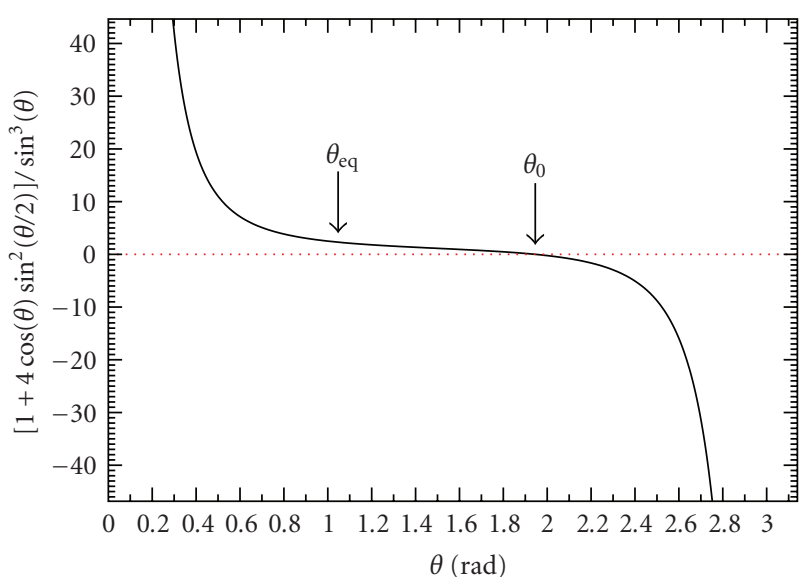

Figure 18: Angular dependency of the isosceles bispectrum as a function of the angle $\theta$ in between the wavevectors $\mathbf{k}_{1}$ and $\mathbf{k}_{2}$. The particular values $\theta_{\mathrm{eq}}=\pi / 3$ corresponds to the equilateral configuration and $\theta_{0}$ makes the bispectrum vanishing. Notice the amplification for flat triangle configurations at $\theta \rightarrow 0$ (squeezed) and $\theta \rightarrow \pi$ (collapsed).

4.7.2. Numerical Results. The previous analytical results can be compared to the $\mathrm{CMB}$ temperature bispectrum derived from the simulated maps of Section 4.2. Numerically, one can use the scale convolution method introduced in [213, 214] and applied to the string bispectrum in [212]. This method relies on the choice of unity window functions in Fourier space $W_{u}(l)$ peaked around a particular wavenumber $u$. Defining

$$
\Theta_{u}(\mathbf{x}) \equiv \int \frac{\mathrm{d} \mathbf{l}}{(2 \pi)^{2}} \widehat{\Theta}_{\mathbf{l}} W_{u}(l) e^{-\mathrm{il} \cdot \mathbf{x}}
$$

one can construct an estimator of the three point function in Fourier space by remarking that

$$
\begin{aligned}
& \int \Theta_{k_{1}}(\mathbf{x}) \Theta_{k_{2}}(\mathbf{x}) \Theta_{k_{3}}(\mathbf{x}) \mathrm{d} \mathbf{x} \\
& =\int \frac{\mathrm{d} \mathbf{p d} \mathbf{q} \mathbf{d} \mathbf{k}}{(2 \pi)^{6}} \widehat{\Theta}_{\mathbf{p}} \widehat{\Theta}_{\mathbf{q}} \widehat{\Theta}_{\mathbf{k}} W_{k_{1}}(p) W_{k_{2}}(q) W_{k_{3}}(k)(2 \pi)^{2} \\
& \quad \times \delta(\mathbf{p}+\mathbf{q}+\mathbf{k}) .
\end{aligned}
$$

For thin enough window functions, $\widehat{\Theta}_{\mathbf{k}}$ remains constant over the window function width and we construct our reduced bispectrum estimator as

$$
b_{k_{1} k_{2} k_{3}}=\frac{1}{S_{k_{1} k_{2} k_{3}}^{(w)}}\left\langle\int \Theta_{k_{1}}(\mathbf{x}) \Theta_{k_{2}}(\mathbf{x}) \Theta_{k_{3}}(\mathbf{x}) \mathrm{d} \mathbf{x}\right\rangle .
$$

The function $S^{(w)}$ is the flat sky equivalent of the inverse Wigner- $3 j$ symbols and reads

$$
S_{k_{1} k_{2} k_{3}}^{(w)}=\int \frac{\mathrm{d} \mathbf{p d} \mathbf{q}}{(2 \pi)^{4}} W_{k_{1}}(p) W_{k_{2}}(q) W_{k_{3}}(|\mathbf{p}+\mathbf{q}|) .
$$

For the window functions such that $W_{u}(k)=1$ for $u-w / 2<$ $k<u+w / 2$, one can approximate

$$
W_{u}(k) \simeq w \delta(k-u),
$$

for small enough values of $w$ compared to the wavenumber $k$. In this case, (87) can be worked out into

$$
S^{(w)} \simeq\left(\frac{w}{2 \pi}\right)^{3} \frac{4 k_{1} k_{2} k_{3}}{\sqrt{\left[\left(k_{1}+k_{2}\right)^{2}-k_{3}^{2}\right]\left[k_{3}^{2}-\left(k_{1}-k_{2}\right)^{2}\right]}} .
$$

In the left panel of Figure 19, we have plotted the mean string bispectrum and its standard deviation obtained by this method over the 300 string CMB maps. For illustration purpose, this plot is for the isosceles configuration having $\theta=0.2$ radians. The right frame of Figure 19 shows the same mean bispectrum but multiplied by $\theta^{3}$, for various small values of $\theta$. As expected from the analytical results, we recover the $1 / \theta^{3}$ behaviour. The wavenumber dependency also matches with the analytical calculations, up to similar slight power differences as we found for the power spectrum. A power law fit against the mean numerical estimator gives

$$
[\ell(\ell+1)]^{3 / 2} b_{\ell \ell \theta} \propto \ell^{-q}, \quad \text { with } q \simeq 2.8,
$$

while the overall amplitude can be evaluated around the minimum variance multipole. At $\ell=5000$, one gets

$$
\left.\left[\frac{\ell(\ell+1)}{2 \pi}\right]^{3 / 2} b_{\ell \ell \theta}\right|_{\ell=5000} \simeq(-2.7 \pm 1.4) \times 10^{-3}\left(\frac{G U}{\theta}\right)^{3}
$$

which also matches with (83) under some crude estimation of the string parameters [212]. Finally, as suggested by Figure 18, the string bispectrum is mostly negative. Integrated over all possible configurations, one recovers the mean negative sample skewness of (53), thereby explaining its origin as a direct consequence of the breaking of the time reversal symmetry in FLRW spacetimes.

4.8. Trispectrum. The trispectrum of the string induced CMB temperature anisotropies can be derived in a similar way. Starting from the definition of the four-point functions

$$
\begin{aligned}
& \left\langle\widehat{\Theta}_{\mathbf{k}_{1}} \widehat{\Theta}_{\mathbf{k}_{2}} \widehat{\Theta}_{\mathbf{k}_{3}} \widehat{\Theta}_{\mathbf{k}_{4}}\right\rangle \\
& =T\left(\mathbf{k}_{1}, \mathbf{k}_{2}, \mathbf{k}_{3}, \mathbf{k}_{4}\right)(2 \pi)^{2} \delta\left(\mathbf{k}_{1}+\mathbf{k}_{2}+\mathbf{k}_{3}+\mathbf{k}_{4}\right),
\end{aligned}
$$

we define the trispectrum as

$$
\begin{aligned}
& T\left(\mathbf{k}_{1}, \mathbf{k}_{2}, \mathbf{k}_{3}, \mathbf{k}_{4}\right) \\
& =\frac{\epsilon^{4}}{\mathcal{A}} \frac{k_{1_{A}} k_{2_{B}} k_{3_{C}} k_{4_{D}}}{k_{1}^{2} k_{2}^{2} k_{3}^{2} k_{4}^{2}} \int \mathrm{d} \sigma_{1} \mathrm{~d} \sigma_{2} \mathrm{~d} \sigma_{3} \mathrm{~d} \sigma_{4}\left\langle\dot{X}_{1}^{A} \dot{X}_{2}^{B} \dot{X}_{3}^{C} \dot{X}_{4}^{D} e^{i \delta^{a b} \mathbf{k}_{a} \cdot \mathbf{X}_{b}}\right\rangle,
\end{aligned}
$$

with $\dot{X}_{a}^{A}=\dot{X}^{A}\left(\sigma_{a}\right),(a, b) \in\{1,2,3,4\}$ and $\mathbf{k}_{1}+\mathbf{k}_{2}+$ $\mathbf{k}_{3}+\mathbf{k}_{4}=0$. (Notice that our denomination "trispectrum" here contains the unconnected part. This one is however non-vanishing only for parallelogram configurations of the wavevectors.) As shown in [215], the trispectrum and the higher $n$-point functions exhibit unfactorable "flat directions" in the $n$-dimensional space of the integration variables $\left\{\sigma_{a}\right\}$. Physically, it means that the leading order part of the 


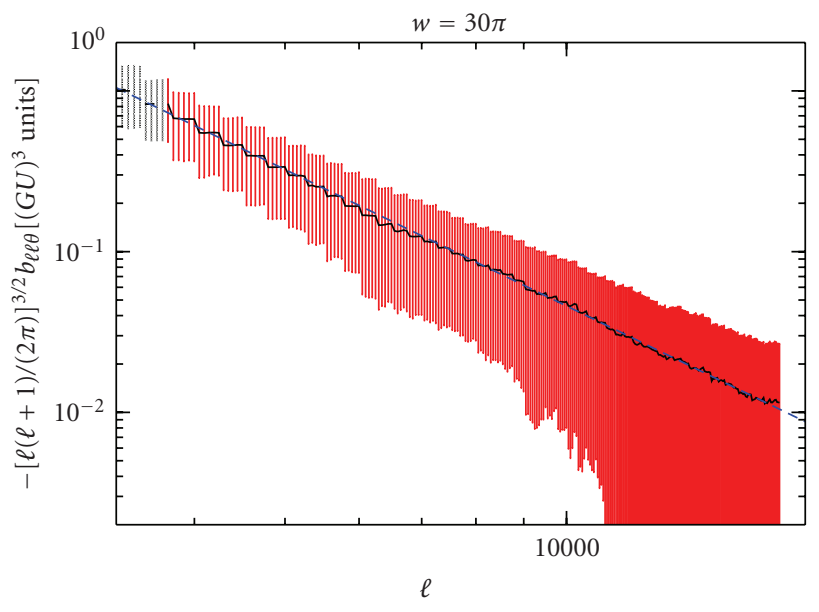

(a)

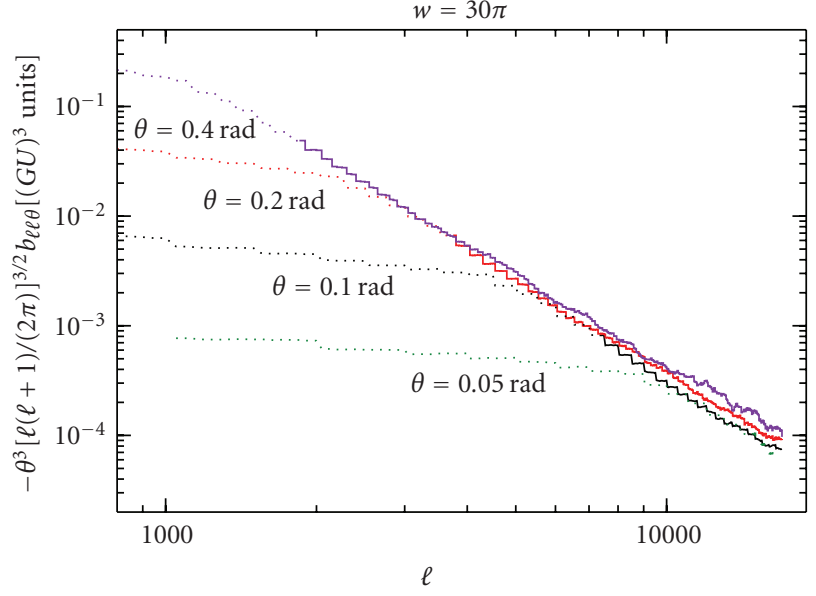

(b)

Figure 19: (a): mean value and standard deviation of the squeezed isosceles bispectrum $[\ell(\ell+1) /(2 \pi)]^{3 / 2} b_{\ell \ell \theta}$ for $\theta=0.2$ radians. The dashed line is the best power law fit. (b) shows its rescaling by $\theta^{3}[\ell(\ell+1) /(2 \pi)]^{3 / 2} b_{\ell \ell \theta}$ showing the $1 / \theta^{3}$ dependency. The spurious plateau (dotted) for the lower multipoles comes from a numerical cutoff associated with the window functions and occurs at $\ell_{\min }(\theta) \simeq 30 \pi /\left(\theta_{\mathrm{fov}} \theta\right)$, the field of view being $\theta_{\text {fov }}=7.2^{\circ}$.

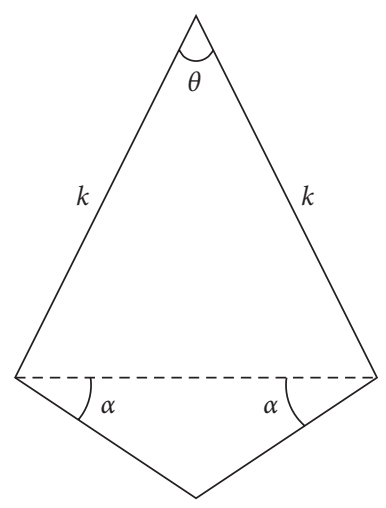

(a)

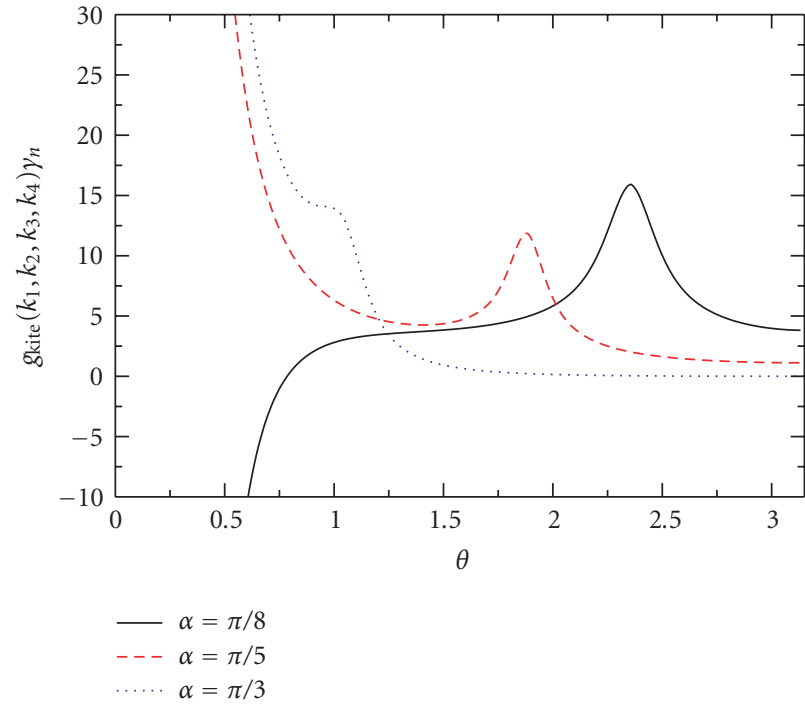

(b)

Figure 20: Trispectrum geometrical factor for the kite quadrilaterals (represented on the left panel) as a function of the opening angle $\theta$, and plotted for various values of $\alpha$. The trispectrum is enhanced in the squeezed limit $\theta \rightarrow 0$. The bump for $\theta_{\mathrm{p}}=\pi-2 \alpha$ corresponds to the parallelogram limit for which the unconnected part is no longer vanishing.

(connected) trispectrum is sensitive to the higher orders of the correlators in (64) to (66). For the trispectrum, the correlator $T(\sigma)$ has to be expanded at next-to-leading order, and following the Polchinski-Rocha model [179], we assume a nonanalytical behaviour for $T(\sigma)$ at small scales

$$
T(\sigma) \simeq \bar{t}^{2}-c_{1}\left(\frac{\sigma}{\hat{\xi}}\right)^{2 \chi}
$$

In the light-cone gauge, we leave $c_{1}$ and $\chi$ as undetermined parameters since they cannot be straightforwardly inferred from the numerics performed in the temporal gauge. Nevertheless, because the correlation should be smaller as $\sigma$ becomes larger, one should have $c_{1}>0$. In the temporal gauge, $\chi$ is directly related to the power law exponent of the scaling loop distribution functions through $\chi=1-p / 2$. As we are bound to show, the mode dependence of the trispectrum will also be uniquely given by this parameter. Once the tangent vector correlator expressed as in (94), the 
integrations in (93) can be performed explicitly, except for parallelogram configurations which have to be dealt with as a special case. After some tedious calculations, an interpolating expression for the trispectrum is [215]

$$
\begin{aligned}
T\left(\mathbf{k}_{1}, \mathbf{k}_{2}, \mathbf{k}_{3}, \mathbf{k}_{4}\right) \simeq & \epsilon^{4} \frac{\bar{v}^{4}}{\bar{t}^{2}} \frac{L \hat{\xi}}{\mathcal{A}}\left(c_{1} \hat{\xi}^{2}\right)^{-1 /(2 \chi+2)} f(\chi) \\
& \times \gamma_{\mathrm{n}}\left(\frac{1}{2 \chi+2}, c_{2} Y^{2} \Lambda^{2 \chi+2}\right) g\left(\mathbf{k}_{1}, \mathbf{k}_{2}, \mathbf{k}_{3}, \mathbf{k}_{4}\right) .
\end{aligned}
$$

In this equation, $f(\chi)$ is a number depending only on the parameter $\chi$

$$
f(\chi)=\frac{\pi}{\chi+1} \Gamma\left(\frac{1}{2 \chi+2}\right)[4(2 \chi+1)(\chi+1)]^{1 /(2 \chi+2)},
$$

while $g\left(\left\{\mathbf{k}_{a}\right\}\right)$ is the trispectrum geometrical factor defined by

$$
g\left(\mathbf{k}_{1}, \mathbf{k}_{2}, \mathbf{k}_{3}, \mathbf{k}_{4}\right)=\frac{\kappa_{12} \kappa_{34}+\kappa_{13} \kappa_{24}+\kappa_{14} \kappa_{23}}{k_{1}^{2} k_{2}^{2} k_{3}^{2} k_{4}^{2}}\left[Y^{2}\right]^{-1 /(2 \chi+2)},
$$

where

$$
Y^{2}\left(\mathbf{k}_{1}, \mathbf{k}_{2}, \mathbf{k}_{3}, \mathbf{k}_{4}\right) \equiv-\kappa_{12}\left(k_{3}^{2} k_{4}^{2}-\kappa_{34}^{2}\right)^{\chi+1}+\circlearrowleft,
$$

and $\circlearrowleft$ stands for cyclic permutations over the indices. The function $\gamma_{\mathrm{n}}(a, x)$ stands for the normalised incomplete lower gamma function defined by

$$
\gamma_{n}(a, x) \equiv \frac{\gamma(a, x)}{\Gamma(a)}=\frac{1}{\Gamma(a)} \int_{0}^{x} t^{a-1} e^{-t} \mathrm{~d} t,
$$

and, finally, $\Lambda$ has been defined by

$$
\begin{aligned}
\Lambda\left(\mathbf{k}_{1}, \mathbf{k}_{2}, \mathbf{k}_{3}, \mathbf{k}_{4}\right) \equiv & \frac{2 L}{\left(k_{1}^{2} k_{2}^{2}-\kappa_{12}^{2}\right)^{1 / 2}+\circlearrowleft} \\
& \times \frac{k_{1} k_{2} k_{3} k_{4}}{\kappa_{12} \kappa_{34}+\kappa_{13} \kappa_{24}+\kappa_{14} \kappa_{23}} .
\end{aligned}
$$

As an application, the trispectrum over parallelogram configurations is obtained when $Y^{2}=0$ and the leading term of (95) simplifies to

$$
T_{0}\left(\mathbf{k}_{1}, \mathbf{k}_{2}, \mathbf{k}_{3}, \mathbf{k}_{4}\right) \simeq \frac{\pi \epsilon^{4} \bar{v}^{4}}{\bar{t}^{2}} \frac{L^{2}}{A k_{1}^{3} k_{2}^{3}|\sin \theta|},
$$

where $\theta$ now refers to the parallelogram angle. Under the scaling transformation $\mathbf{k}_{a} \rightarrow \lambda \mathbf{k}_{a}$, the parallelogram trispectrum scales as

$$
T_{0}\left(\lambda \mathbf{k}_{1}, \lambda \mathbf{k}_{2}, \lambda \mathbf{k}_{3}, \lambda \mathbf{k}_{4}\right)=\lambda^{-6} T_{0}\left(\mathbf{k}_{1}, \mathbf{k}_{2}, \mathbf{k}_{3}, \mathbf{k}_{4}\right) .
$$

For parallelograms, as already mentioned, the trispectrum also gets a contribution from the unconnected part of the four-point function, which is Gaussian and reads

$$
T_{0}^{\mathrm{uc}}\left(\mathbf{k}_{1}, \mathbf{k}_{2}, \mathbf{k}_{3}, \mathbf{k}_{4}\right)=\mathcal{A} P\left(k_{1}\right) P\left(k_{2}\right)+\circlearrowleft .
$$

Using (63), ones sees that the unconnected part also behaves as $\lambda^{-6}$. Therefore, the non-Gaussian contributions for parallelogram configurations remain of the same order of magnitude as the Gaussian ones, with however, and again, an exception in the squeezed limit $\theta \rightarrow 0$.

The most interesting situations come when $Y^{2} \neq 0$. For these quadrilaterals, the Gaussian contribution vanishes and solely a non-Gaussian statistics can source the trispectrum. At large wavenumber (small angles), one has $Y^{2} \gg 1$ such that the normalised lower incomplete gamma function in (95) is close to unity:

$$
\begin{aligned}
& T_{\infty}\left(\mathbf{k}_{1}, \mathbf{k}_{2}, \mathbf{k}_{3}, \mathbf{k}_{4}\right) \\
& \simeq \epsilon^{4} \frac{\bar{v}^{4}}{\bar{t}^{2}} \frac{L \hat{\xi}}{\mathcal{A}}\left(c_{1} \hat{\xi}^{2}\right)^{-1 /(2 \chi+2)} f(\chi) g\left(\mathbf{k}_{1}, \mathbf{k}_{2}, \mathbf{k}_{3}, \mathbf{k}_{4}\right) .
\end{aligned}
$$

Under the scaling transformation $\mathbf{k}_{a} \rightarrow \lambda \mathbf{k}_{a}$, the geometric factor, and thus (104), scales as

$$
g\left(\lambda \mathbf{k}_{1}, \lambda \mathbf{k}_{2}, \lambda \mathbf{k}_{3}, \lambda \mathbf{k}_{4}\right)=\lambda^{-\rho} g\left(\mathbf{k}_{1}, \mathbf{k}_{2}, \mathbf{k}_{3}, \mathbf{k}_{4}\right),
$$

with

$$
\rho=6+\frac{1}{\chi+1}
$$

As claimed, for NG strings, $\rho$ is directly given by the power law of the loop distribution [146]. Since this exponent is different from the one associated with parallelogram configurations it may actually be used to distinguish a trispectrum sourced by cosmic strings with the one generated by other non-Gaussian effects.

In Figure 20, we have plotted the geometrical factor $g\left(\mathbf{k}_{1}, \mathbf{k}_{2}, \mathbf{k}_{2}, \mathbf{k}_{4}\right)$ for the kite quadrilaterals (represented in the same figure), as a function of their opening angles $\theta$ and $\alpha$.

As for the bispectrum, the trispectrum is enhanced on the squeezed configuration obtained when $\theta$ becomes small. In this limit (97) can be expanded as

$$
\begin{aligned}
g \sim & \frac{8 \cos ^{2}(\alpha)}{k^{\rho} \theta^{\rho-3}}(1-2 \cos 2 \alpha) \\
& \times\left[2(1+\chi) \tan ^{2}(\alpha)-1+4^{\chi}\left(1-\tan ^{2} \alpha\right)\right]^{-1 /(2 \chi+2)},
\end{aligned}
$$

and one recovers the mode dependency in $k^{-\rho}$ while the amplitude is amplified as $\theta^{\rho-3}$. As discussed in the previous section, the singular limit $\theta \rightarrow 0$ is never reached with a finite resolution beam.

4.9. Comparison with Data. The cosmic string bispectrum and trispectrum associated with the flat polygonal configurations are the best suited to look for string signatures. However, it is not easy to compare with existing constraints as much of the literature focuses on particular models of primordial non-Gaussianity. For instance, in the local type of primordial non-Gaussianities, the parameter $f_{\mathrm{NL}}$ characterises the primordial bispectrum and maximal amplitude occurs for squeezed triangle configurations, as 
it is the case for the cosmic strings [216]. However, as a result of the CMB transfer functions, a given value of $f_{\mathrm{NL}}$ corresponds to oscillating damped patterns of the $\mathrm{CMB}$ temperature bispectrum, which are completely different of the power laws we have found for the string bispectrum at small scales. The current bounds on $f_{\mathrm{NL}}$ being precisely obtained from template matching procedures, they cannot be applied to the strings [44, 217, 218]. For this reason, the parameters used to quantify primordial non-Gaussianities are not well suited here, precisely because we expect the string non-Gaussianities to be nonprimordial. An efficient approach would be to use a template matching procedure with the formulae derived in the previous sections. Another approach might be to estimate what values the primordial parameters, such as $f_{\mathrm{NL}}$ and $\tau_{\mathrm{NL}}$, would assume if the non-Gaussianities were actually due to strings. Notice that asking such a question would be close to find the best amplitude of a sine function to fit a power law. However, since primordial non-Gaussianities are and will be tested in CMB data anyway, one could answer this question by performing a Fisher matrix analysis along the lines of $[55,57]$.

\section{Conclusion and Perspectives}

The results presented in this paper were essentially concerned with Nambu-Goto type of cosmic strings, which is the simplest realisation of a one-dimensional spatially extended object. As a result, they should not be blindly extrapolated to other types of string, although, as argued in Section 2, some of them are expected to be generic. In particular, due to the scaling of the long strings, cosmic string loops do not influence significantly the $\mathrm{CMB}$ observables. Changing the intercommuting probability is expected to rescale some of the presented results $[219,220]$, but in a way which remains to be quantified.

In Section 3, we have briefly reviewed the current understanding of the cosmological evolution of a string network by means of FLRW numerical simulations, which is a nontrivial problem even for NG strings. Observable predictions crucially depend on this step. Numerical simulations can be avoided by making some assumptions on the string distribution but at the expense of introducing unnecessary extra parameters. When approximate analytical models are then used to derive observable predictions, one should keep in mind that the results are as uncertain as the values assumed for the additional parameters. Provided one is interested in length scales not affected by gravitational back-reaction effects, all of the statistical properties of an NG string network in scaling depends only on one unknown physical parameter: the string energy density per unit length $U$, not more.(The expansion rate is supposed to be known.)

In this context, Section 4 discusses the non-Gaussian effects induced by a cosmological string network in the $\mathrm{CMB}$ temperature anisotropies. We have shown that string induced CMB fluctuations have a negative skewness and a non-vanishing kurtosis. On a CMB temperature anisotropies map, these non-Gaussianities imprint characteristic signatures in a multifractal analysis as well as in the gradient magnitude, both being more significant at small angles. This property is recovered in Fourier space: the CMB angular power spectrum decays at most as $1 / \ell$, for the large multipoles $\ell$, and strings become the dominant sources of primary fluctuations. The skewness appears to be the direct consequence of the breaking of the time reversal symmetry in an expanding universe, and implies the existence of a non-vanishing bispectrum. Using analytical approximations, tested and confirmed by numerical simulations, we then derived the expected bispectrum and trispectrum of string induced CMB temperature anisotropies for the large multipoles. Although the bispectrum decays not faster than $\ell^{-6}$, the trispectrum multipole dependency is in $\ell^{-\rho}$, where $\rho=$ $6+1 /(\chi+1)$ and $\chi$ is a small number related to the tangent vector correlator and the NG loop distribution. Due to the line-like $\mathrm{CMB}$ patterns induced by the strings, both the bispectrum and trispectrum are enhanced on all elongated triangle and quadrilateral configurations of the wavevectors. These ones may constitute the best configurations to look for a non-Gaussian string signal while being experimentally limited by finite beam resolution. Let us note that our expressions have been derived in the flat sky approximation. String non-Gaussianities at small multipoles is still an open problem which could be dealt with full sky string maps [201, 221, 222]. However, if, as the current constraints suggest, cosmic strings marginally contribute to the large-scale CMB anisotropies, then they should show up at large multipoles in all of the above mentioned observables. This is precisely where the experimental efforts are directed. At very small angular scales, the difficulties will certainly be to separate the string signals from the astrophysical sources. Interestingly, the very soon accessible intermediate angles probed by the Planck satellite, and the other ground-based telescopes, may not suffer from this problem and could be an open window on cosmic strings.

\section{Acknowledgments}

It is a pleasure to thank Patrick Peter, Mairi Sakellariadou, Danielle Steer and Teruaki Suyama for a careful reading of the paper and their enlightening comments. This paper is supported by the Belgian Federal Office for Scientific, Technical and Cultural Affairs, under the Inter-University Attraction Pole Grant P6/11.

\section{References}

[1] D. A. Kirzhnits and A. D. Linde, "Macroscopic consequences of the Weinberg model," Physics Letters B, vol. 42, no. 4, pp. 471-474, 1972.

[2] I. Y. Kobsarev, L. B. Okun, and Y. B. Zeldovich, "Spontaneus CP-violation and cosmology," Physics Letters B, vol. 50, no. 3, pp. 340-342, 1974.

[3] T. W. B. Kibble, "Topology of cosmic domains and strings," Journal of Physics A, vol. 9, no. 8, pp. 1387-1398, 1976.

[4] M. B. Hindmarsh and T. W. B. Kibble, "Cosmic strings," Reports on Progress in Physics, vol. 58, no. 5, pp. 477-562, 1995. 
[5] A. Vilenkin and E. P. S. Shellard, Cosmic Strings and Other Topological Defects, Cambridge University Press, Cambridge, UK, 2000.

[6] M. Sakellariadou, "Cosmic strings," Lecture Notes in Physics, vol. 718, pp. 247-288, 2007.

[7] P. Peter and J.-P. Uzan, Primordial Cosmology, Oxford Graduate Texts, Oxford University Press, Oxford, UK, 2009.

[8] S. Sarkar, "Big bang nucleosynthesis and physics beyond the standard model," Reports on Progress in Physics, vol. 59, no. 12, pp. 1493-1609, 1996.

[9] R. H. Cyburt, B. D. Fields, K. A. Olive, and E. Skillman, "New BBN limits on physics beyond the standard model from ${ }^{4} \mathrm{He}$," Astroparticle Physics, vol. 23, no. 3, pp. 313-323, 2005.

[10] T. W. B. Kibble, G. Lazarides, and Q. Shafi, "Walls bounded by strings," Physical Review D, vol. 26, no. 2, pp. 435-439, 1982.

[11] P. Langacker and S.-Y. Pi, "Magnetic monopoles in grand unified theories," Physical Review Letters, vol. 45, no. 1, pp. $1-4,1980$.

[12] A. H. Guth, "Inflationary universe: a possible solution to the horizon and flatness problems," Physical Review D, vol. 23, no. 2, pp. 347-356, 1981.

[13] A. A. Starobinsky, "A new type of isotropic cosmological models without singularity," Physics Letters B, vol. 91, no. 1, pp. 99-102, 1980.

[14] A. D. Linde, "A new inflationary universe scenario: a possible solution of the horizon, flatness, homogeneity, isotropy and primordial monopole problems," Physics Letters B, vol. 108, no. 6, pp. 389-393, 1982.

[15] A. A. Starobinsky, "Dynamics of phase transition in the new inflationary universe scenario and generation of perturbations," Physics Letters B, vol. 117, no. 3-4, pp. 175-178, 1982.

[16] V. F. Mukhanov, H. A. Feldman, and R. H. Brandenberge, "Theory of cosmological perturbations, part I: classical perturbations," Physics Reports, vol. 215, no. 5-6, pp. 203256, 1992.

[17] V. F. Mukhanov, H. A. Feldman, and R. H. Brandenberge, "Theory of cosmological perturbations, part II: quantum perturbations," Physics Reports, vol. 215, no. 5-6, pp. 257$295,1992$.

[18] V. F. Mukhanov, H. A. Feldman, and R. H. Brandenberge, "Theory of cosmological perturbations, part III: extensions," Physics Reports, vol. 215, no. 5-6, pp. 296-333, 1992.

[19] J. Martin and C. Ringeval, "Inflation after WMAP3: confronting the slow-roll and exact power spectra with $\mathrm{CMB}$ data," Journal of Cosmology and Astroparticle Physics, vol. 2006, no. 8, article 9, 2006.

[20] P. P. Avelino, C. J. A. P. Martins, and E. P. S. Shellard, "Effects of inflation on a cosmic string loop population," Physical Review D, vol. 76, no. 8, Article ID 083510, 7 pages, 2007.

[21] R. Jeannerot, J. Rocher, and M. Sakellariadou, "How generic is cosmic string formation in supersymmetric grand unified theories," Physical Review D, vol. 68, no. 10, Article ID 103514, 20 pages, 2003.

[22] J. Rocher and M. Sakellariadou, " $D$-term inflation, cosmic strings, and consistency with cosmic microwave background measurements," Physical Review Letters, vol. 94, no. 1, Article ID 011303, 2005.

[23] J. Rocher and M. Sakellariadou, " $D$-term inflation in nonminimal supergravity," Journal of Cosmology and Astroparticle Physics, no. 11, article 1, 2006.

[24] R. A. Battye, B. Garbrecht, and A. Moss, "Constraints on supersymmetric hybrid inflation models," Journal of
Cosmology and Astroparticle Physics, vol. 2006, no. 9, article 7, 2006.

[25] K. Becker, M. Becker, and J. H. Schwarz, String Theory and MTheory: A Modern Introduction, Cambridge University Press, Cambridge, UK, 2007.

[26] G. Dvali and S.-H. H. Tye, "Brane inflation," Physics Letters $B$, vol. 450, no. $1-3$, pp. 72-82, 1999.

[27] S. H. S. Alexander, "Inflation from $D-\bar{D}$ brane annihilation," Physical Review D, vol. 65, no. 2, Article ID 023507, 2002.

[28] S. Kachru, R. Kallosh, A. Linde, J. Maldacena, L. McAllister, and S. P. Trivedi, "Towards inflation in string theory," Journal of Cosmology and Astroparticle Physics, vol. 2003, no. 10, article 13, 2003.

[29] C. P. Burgess, M. Majumdar, D. Nolte, F. Quevedo, G. Rajesh, and R.-J. Zhang, "The inflationary brane-antibrane universe," Journal of High Energy Physics, vol. 5, no. 7, article 47, 2001.

[30] S. Sarangi and S.-H. Tye, "Cosmic string production towards the end of brane inflation," Physics Letters B, vol. 536, no. 3-4, pp. 185-192, 2002.

[31] G. Dvali and A. Vilenkin, "Formation and evolution of cosmic D strings," Journal of Cosmology and Astroparticle Physics, vol. 2004, no. 3, article 10, 2004.

[32] N. T. Jones, H. Stoica, and S.-H. H. Tye, "The production, spectrum and evolution of cosmic strings in brane inflation," Physics Letters B, vol. 563, no. 1-2, pp. 6-14, 2003.

[33] A.-C. Davis and T. W. B. Kibble, "Fundamental cosmic strings," Contemporary Physics, vol. 46, no. 5, pp. 313-322, 2005.

[34] E. J. Copeland and T. W. B. Kibble, "Cosmic strings and superstrings," Proceedings of the Royal Society A, vol. 466, no. 2115, pp. 623-657, 2010.

[35] M. Sakellariadou, "Cosmic superstrings," Philosophical Transactions of the Royal Society A, vol. 366, no. 1877, pp. 2881-2894, 2008.

[36] M. Sakellariadou, "Cosmic strings and cosmic superstrings," Nuclear Physics B-Proceedings Supplements, vol. 192-193, pp. 68-90, 2009.

[37] F. R. Bouchet, D. P. Bennett, and A. Stebbins, "Patterns of the cosmic microwave background from evolving string networks," Nature, vol. 335, no. 6189, pp. 410-414, 1988.

[38] B. Carter, "Duality relation between charged elastic strings and superconducting cosmic strings," Physics Letters B, vol. 224, no. 1-2, pp. 61-66, 1989.

[39] R. Durrer, M. Kunz, and A. Melchiorri, "Cosmic structure formation with topological defects," Physics Report, vol. 364, no. 1, pp. 1-81, 2002.

[40] R. Durrer, A. Gangui, and M. Sakellariadou, "Doppler peaks in the angular power spectrum of the cosmic microwave background: a fingerprint of topological defects," Physical Review Letters, vol. 76, no. 4, pp. 579-582, 1996.

[41] J. Magueijo, A. Albrecht, P. Ferreira, and D. Coulson, "The structure of Doppler peaks induced by active perturbations," Physical Review D, vol. 54, no. 6, pp. 3727-3744, 1996.

[42] A. Albrecht, R. A. Battye, and J. Robinson, "The case against scaling defect models of cosmic structure formation," Physical Review Letters, vol. 79, no. 24, pp. 4736-4739, 1997.

[43] D. N. Spergel, R. Bean, O. Doré et al., "Three-year Wilkinson Microwave Anisotropy Probe (WMAP) observations: implications for cosmology," Astrophysical Journal, Supplement Series, vol. 170, no. 2, pp. 377-408, 2007.

[44] E. Komatsu, J. Dunkley, M. R. Nolta et al., "Five-year wilkinson microwave anisotropy probe observations: cosmological 
interpretation," Astrophysical Journal, Supplement Series, vol. 180, no. 2, pp. 330-376, 2009.

[45] F. R. Bouchet, P. Peter, A. Riazuelo, and M. Sakellariadou, "Evidence against or for topological defects in the BOOMERanG data?" Physical Review D, vol. 65, no. 2, Article ID 021301, 4 pages, 2001.

[46] A. A. Fraisse, "Limits on defects formation and hybrid inflationary models with three-year WMAP observations," Journal of Cosmology and Astroparticle Physics, vol. 2007, no. 3, article 8, 2007.

[47] N. Bevis, M. Hindmarsh, M. Kunz, and J. Urrestilla, "Fitting cosmic microwave background data with cosmic strings and inflation," Physical Review Letters, vol. 100, no. 2, Article ID 021301, 2008.

[48] A. M. Gilbert and L. Perivolaropoulos, "Spectra and statistics of cosmic string perturbations on the microwave background: a Monte Carlo approach," Astroparticle Physics, vol. 3, no. 3, pp. 283-294, 1995.

[49] E. Jeong and G. F. Smoot, "The validity of the cosmic string pattern search with the cosmic microwave background," Astrophysical Journal, vol. 661, no. 1, pp. L1-L4, 2007.

[50] S. Amsel, J. Berger, and R. H. Brandenberger, "Detecting cosmic strings in the CMB with the canny algorithm," Journal of Cosmology and Astroparticle Physics, vol. 2008, no. 4, article $15,2008$.

[51] A. Gangui, L. Pogosian, and S. Winitzki, "CMB bispectrum from active models of structure formation," Physical Review D, vol. 64, no. 4, Article ID 043001, 7 pages, 2001.

[52] L. Pogosian and M. Wyman, "B-modes from cosmic strings," Physical Review D, vol. 77, no. 8, Article ID 083509, 11 pages, 2008.

[53] J. R. Gott III, "Gravitational lensing effects of vacuum strings-exact solutions," Astrophysical Journal, vol. 288, no. 8, pp. 422-427, 1985.

[54] N. Kaiser and A. Stebbins, "Microwave anisotropy due to cosmic strings," Nature, vol. 310, no. 5976, pp. 391-393, 1984.

[55] E. Sefusatti and E. Komatsu, "The bispectrum of galaxies from high-redshift galaxy surveys: primordial nonGaussianity and non-linear galaxy bias," Physical Review D, vol. 76, no. 8, Article ID 083004, 17 pages, 2007.

[56] C. Pitrou, J.-P. Uzan, and F. Bernardeau, "Cosmic microwave background bispectrum on small angular scales," Physical Review D, vol. 78, no. 6, Article ID 063526, 2008.

[57] D. Nitta, E. Komatsu, N. Bartolo, S. Matarrese, and A. Riotto, "CMB anisotropies at second order III: bispectrum from products of the first-order perturbations," Journal of Cosmology and Astroparticle Physics, vol. 2009, no. 5, article $14,2009$.

[58] L. Boubekeur, P. Creminelli, G. D’Amico, J. Norea, and F. Vernizzi, "Sachs-wolfe at second order: the CMB bispectrum on large angular scales," Journal of Cosmology and Astroparticle Physics, vol. 2009, no. 8, article 29, 2009.

[59] S. Weinberg, "Conceptual foundations of the unified theory of weak and electromagnetic interactions," Reviews of Modern Physics, vol. 52, no. 3, pp. 515-523, 1980.

[60] A. J. Gill and R. J. Rivers, "Dynamics of vortex and monopole production by quench-induced phase separation," Physical Review D, vol. 51, no. 12, pp. 6949-6958, 1995.

[61] G. Karra and R. J. Rivers, "Initial vortex densities after a temperature quench," Physics Letters B, vol. 414, no. 1-2, pp. 28-33, 1997.

[62] L. M. A. Bettencourt, T. S. Evans, and R. J. Rivers, "Winding number correlation functions and cosmic string formation," Physical Review D, vol. 53, no. 2, pp. 668-680, 1996.

[63] E. Kavoussanaki, R. Monaco, and R. J. Rivers, "Testing the kibble-zurek scenario with annular Josephson tunnel junctions," Physical Review Letters, vol. 85, no. 16, pp. 34523455, 2000.

[64] N. K. Nielsen and P. Olesen, "Dynamical properties of superconducting cosmic strings," Nuclear Physics B, vol. 291, pp. 829-846, 1987.

[65] P. Peter, "Superconducting cosmic string: equation of state for spacelike and timelike current in the neutral limit," Physical Review D, vol. 45, no. 4, pp. 1091-1102, 1992.

[66] C. Ringeval, "Equation of state of cosmic strings with fermionic current carriers," Physical Review D, vol. 63, no. 6, Article ID 063508, 24 pages, 2001.

[67] A. Vilenkin, "Gravitational field of vacuum domain walls and strings," Physical Review D, vol. 23, no. 4, pp. 852-857, 1981.

[68] L. M. A. Bettencourt and R. J. Rivers, "Interactions between U(1) cosmic strings: an analytical study," Physical Review D, vol. 51, no. 4, pp. 1842-1853, 1995.

[69] N. D. Antunes, L. M. A. Bettencourt, and M. Hindmarsh, "Thermodynamics of cosmic string densities in U(1) scalar field theory," Physical Review Letters, vol. 80, no. 5, pp. 908911, 1998.

[70] T. Vachaspati and A. Vilenkin, "Formation and evolution of cosmic strings," Physical Review D, vol. 30, no. 10, pp. 20362045, 1984

[71] M. Hindmarsh and A. Rajantie, "Phase transition dynamics in the hot Abelian Higgs model," Physical Review D, vol. 64, no. 6, Article ID 065016, 13 pages, 2001.

[72] M. Donaire and A. Rajantie, "Heavy cosmic strings," Physical Review D, vol. 73, no. 6, Article ID 063517, 4 pages, 2006.

[73] A. Rajantie, "Super-horizon cosmic string correlations," Physical Review D, vol. 79, no. 4, Article ID 043515, 2009.

[74] M. Sakellariadou and H. Stoica, "Dynamics of F/D networks: the role of bound states," Journal of Cosmology and Astroparticle Physics, vol. 2008, no. 8, article 38, 2008.

[75] G. Vincent, N. D. Antunes, and M. Hindmarsh, "Numerical simulations of string networks in the Abelian-Higgs model," Physical Review Letters, vol. 80, no. 11, pp. 2277-2280, 1998.

[76] J. N. Moore, E. P. S. Shellard, and C. J. A. P. Martins, "Evolution of Abelian-Higgs string networks," Physical Review D, vol. 65, no. 2, Article ID 023503, 19 pages, 2001.

[77] M. Hindmarsh, S. Stuckey, and N. Bevis, "Abelian Higgs cosmic strings: small-scale structure and loops," Physical Review D, vol. 79, no. 12, Article ID 123504, 2009.

[78] R. L. Davis, "Goldstone bosons in string models of galaxy formation," Physical Review D, vol. 32, no. 12, pp. 3172-3177, 1985.

[79] R. Durrer, M. Kunz, and A. Melchiorri, "Cosmic microwave background anisotropies from scaling seeds: global defect models," Physical Review D, vol. 59, no. 12, Article ID 123005, 26 pages, 1999.

[80] M. Yamaguchi, "Scaling property of the global string in the radiation dominated universe," Physical Review D, vol. 60, no. 10, Article ID 103511, 10 pages, 1999.

[81] M. Yamaguchi, J. Yokoyama, and M. Kawasaki, "Evolution of a global string network in a matter-dominated universe," Physical Review D, vol. 61, no. 6, Article ID 061301, 5 pages, 2000.

[82] P. McGraw, "Evolution of a non-abelian cosmic string network," Physical Review D, vol. 57, no. 6, pp. 3317-3339, 1998. 
[83] A. Vilenkin, "String-dominated universe," Physical Review Letters, vol. 53, no. 10, pp. 1016-1018, 1984.

[84] G. Dvali and G. Senjanović, "Flavor changing strings and domain walls," Physical Review Letters, vol. 72, no. 1, pp. 912, 1994.

[85] D. Spergel and U.-L. Pen, "Cosmology in a string-dominated universe," Astrophysical Journal, vol. 491, no. 2, pp. L67-L71, 1997.

[86] M. Bucher and D. Spergel, "Is the dark matter a solid?" Physical Review D, vol. 60, no. 4, Article ID 043505, 11 pages, 1999.

[87] K. Hashimoto and D. Tong, "Reconnection of non-abelian cosmic strings," Journal of Cosmology and Astroparticle Physics, vol. 2005, no. 9, article 4, pp. 53-72, 2005.

[88] M. Eto, et al., "Universal reconnection of non-Abelian cosmic strings," Physics Review Letters, vol. 98, no. 9, Article ID 091602, 4 pages, 2007.

[89] M. Eto, Y. Isozumi, M. Nitta, K. Ohashi, and N. Sakai, "Solitons in the Higgs phase: The moduli matrix approach," Journal of Physics A, vol. 39, no. 26, pp. R315-R392, 2006.

[90] M. Shifman and A. Yung, "Supersymmetric solitons and how they help us understand and non-abelian gauge theories," Reviews of Modern Physics, vol. 79, no. 4, pp. 1139-1196, 2007.

[91] M. Kobayashi, Y. Kawaguchi, M. Nitta, and M. Ueda, "Collision dynamics and rung formation of non-Abelian vortices," Physics Review Letters, vol. 103, no. 11, Article ID 115301, 4 pages, 2009.

[92] T. Vachaspati and A. Achúcarro, "Semilocal cosmic strings," Physical Review D, vol. 44, no. 10, pp. 3067-3071, 1991.

[93] A. Achúcarro, J. Borrill, and A. R. Liddle, "Formation rate of semilocal strings," Physical Review Letters, vol. 82, no. 19, pp. 3742-3745, 1999.

[94] A. Achúcarro and T. Vachaspati, "Semilocal and electroweak strings," Physics Report, vol. 327, no. 6, pp. 347-426, 2000.

[95] M. Hindmarsh, "Existence and stability of semilocal strings," Physical Review Letters, vol. 68, no. 9, pp. 1263-1266, 1992.

[96] X. Zhang, T. Huang, and R. H. Brandenberger, "Pion and $\eta^{\prime}$ strings," Physical Review D, vol. 58, no. 2, Article ID 027702, 3 pages, 1998.

[97] A. P. Balachandran and S. Digal, "Topological string defect formation during the chiral phase transition," International Journal of Modern Physics A, vol. 17, no. 8, pp. 1149-1158, 2002.

[98] K. B. W. Buckley and A. R. Zhitnitsky, "Superconducting K strings in high density QCD," Journal of High Energy Physics, vol. 6, no. 8, pp. 235-249, 2002.

[99] R. H. Brandenberger, B. Carter, and A.-C. Davis, "Microwave background constraints on decaying defects," Physics Letters $B$, vol. 534, no. 1-4, pp. 1-7, 2002.

[100] M. Nitta and N. Shiiki, "Non-Abelian global strings at chiral phase transition," Physics Letters B, vol. 658, no. 4, pp. 143$147,2008$.

[101] M. Eto, E. Nakano, and M. Nitta, "Non-Abelian global vortices," Nuclear Physics B, vol. 821, no. 1-2, pp. 129-150, 2009.

[102] E. Nakano, M. Nitta, and T. Matsuura, "Non-Abelian strings in hot or dense QCD," Progress of Theoretical Physics Supplement, vol. 174, pp. 254-257, 2008.

[103] M. Eto, M Nitta, and N. Yamamoto, "Instabilities of nonnbelian vortices in dense QCD," Physical Review Letters, vol. 104, no. 16, Article ID 161601, 4 pages, 2010.

[104] E. Babichev, "Global topological k-defects," Physical Review $D$, vol. 74, no. 8, Article ID 085004, 7 pages, 2006.
[105] E. Babichev, "Gauge k-vortices," Physical Review D, vol. 77, no. 6, Article ID 065021, 2008.

[106] S. Sarangi, "DBI global strings,” Journal of High Energy Physics, vol. 2008, no. 7, article 18, 2008.

[107] E. Babichev, P. Brax, C. Caprini, J. Martin, and D. A. Steer, "Dirac Born Infeld (DBI) cosmic strings," Journal of High Energy Physics, vol. 2009, no. 3, article 91, 2009.

[108] E. Witten, "Superconducting strings," Nuclear Physics B, vol. 249, no. 4, pp. 557-592, 1985.

[109] B. Carter, "Essentials of classical brane dynamics," International Journal of Theoretical Physics, vol. 40, no. 12, pp. 20992130, 2001.

[110] R. L. Davis, "Semitopological solitons," Physical Review D, vol. 38, no. 12, pp. 3722-3730, 1988.

[111] R. Brandenberger, B. Carter, A.-C. Davis, and M. Trodden, "Cosmic vortons and particle physics constraints," Physical Review D, vol. 54, no. 10, pp. 6059-6071, 1996.

[112] C. Ringeval, "Fermionic massive modes along cosmic strings," Physical Review D, vol. 64, no. 12, Article ID 123505, 2001.

[113] B. Carter and X. Martin, "Dynamical instability criterion for circular (vorton) string loops," Annals of Physics, vol. 227, no. 1, pp. 151-171, 1993.

[114] X. Martin, "Zones of dynamical instability for rotating string loops," Physical Review D, vol. 50, no. 12, pp. 7479-7492, 1994.

[115] J. Polchinski, "Introduction to cosmic F- and D-strings," in Lectures Given at NATO Advanced Study Institute and EC Summer School on String Theory: From Gauge Interactions to Cosmology, pp. 229-253, Cargese, France, June 2004.

[116] A.-C. Davis, P. Brax, and C. Van De Bruck, "Brane inflation and defect formation," Philosophical Transactions of the Royal Society A, vol. 366, no. 1877, pp. 2833-2842, 2008.

[117] E. Witten, “Cosmic superstrings," Physics Letters B, vol. 153, no. 4-5, pp. 243-246, 1985.

[118] L. Lorenz, J. Martin, and C. Ringeval, "Constraints on kinetically modified inflation from WMAP5," Physical Review D, vol. 78, no. 6, Article ID 063543, 2008.

[119] S. B. Giddings, S. Kachru, and J. Polchinski, "Hierarchies from fluxes in string compactifications," Physical Review D, vol. 66, no. 10, Article ID 106006, 2002.

[120] I. R. Klebanov and M. J. Strassler, "Supergravity and a confining gauge theory: duality cascades and $\chi \mathrm{SB}$-resolution of naked singularities," Journal of High Energy Physics, vol. 4, no. 8, pp. 21-35, 2000.

[121] L. Lorenz, J. Martin, and C. Ringeval, "Brane inflation and the WMAP data: a Bayesian analysis," Journal of Cosmology and Astroparticle Physics, vol. 2008, no. 4, article 1, 2008.

[122] H. Firouzjahi and S.-H. H. Tye, "Brane inflation and cosmic string tension in superstring theory," Journal of Cosmology and Astroparticle Physics, vol. 2005, no. 3, pp. 115-131, 2005.

[123] S. S. Gubser, C. P. Herzog, and I. R. Klebanov, "Symmetry breaking and axionic strings in the warped deformed conifold," Journal of High Energy Physics, vol. 8, no. 9, pp. 795820, 2004.

[124] H. Firouzjahi, L. Leblond, and S.-H. H. Tye, "The $(p, q)$ string tension in a warped deformed conifold," Journal of High Energy Physics, vol. 2006, no. 5, article 47, 2006.

[125] M. G. Jackson, "A note on Cosmic $(p, q, r)$ strings," Physical Review D, vol. 75, no. 8, Article ID 087301, 2007.

[126] E. J. Copeland, T. W. B. Kibble, and D. A. Steer, "Constraints on string networks with junctions," Physical Review D, vol. 75, no. 6, Article ID 065024, 12 pages, 2007. 
[127] R. J. Rivers and D. A. Steer, "Statistical mechanics of strings with Y-junctions," Physical Review D, vol. 78, no. 2, Article ID 023521, 13 pages, 2008.

[128] N. Bevis, E. J. Copeland, P.-Y. Martin, et al., "Evolution and stability of cosmic string loops with Y-junctions," Physical Review D, vol. 80, no. 12, Article ID 125030, 14 pages, 2009.

[129] P. M. Saffin, "A practical model for cosmic $(p, q)$ superstrings," Journal of High Energy Physics, vol. 2005, no. 9, pp. 283-295, 2005.

[130] I. Cho, Y. Kim, and B. Kyae, "DF-strings from $D 3 \bar{D} 3$ as cosmic strings," Journal of High Energy Physics, vol. 2006, no. 4, article 12, 2006.

[131] M. Hindmarsh and P. M. Saffin, "Scaling in a $S U(2) / \mathbb{Z} 3$ model of cosmic superstring networks," Journal of High Energy Physics, vol. 2006, no. 8, article 66, 2006.

[132] A. Rajantie, M. Sakellariadou, and H. Stoica, "Numerical experiments with $p \mathrm{~F}$ - and $p$ D-strings: the formation of $(p, q)$ bound states," Journal of Cosmology and Astroparticle Physics, vol. 2007, no. 11, article 21, 2007.

[133] B. Carter, "Stability and characteristic propagation speeds in superconducting cosmic and other string models," Physics Letters B, vol. 228, no. 4, pp. 466-470, 1989.

[134] B. Carter, "Outer curvature and conformal geometry of an imbedding," Journal of Geometry and Physics, vol. 8, pp. 53$88,1992$.

[135] R. M. Wald, General Relativity, University of Chicago Press, Chicago, Ill, USA, 1984.

[136] B. Carter, M. Sakellariadou, and X. Martin, "Cosmological expansion and thermodynamic mechanisms in cosmic string dynamics," Physical Review D, vol. 50, no. 2, pp. 682-699, 1994.

[137] B. Carter, "Integrable equation of state for noisy cosmic string," Physical Review D, vol. 41, no. 12, pp. 3869-3872, 1990.

[138] B. Carter, "Transonic elastic model for wiggly Goto-Nambu string," Physical Review Letters, vol. 74, no. 16, pp. 30983101, 1995.

[139] B. Carter and D. A. Steer, "Symplectic structure for elastic and chiral conducting cosmic string models," Physical Review D, vol. 69, no. 12, Article ID 125002, 9 pages, 2004.

[140] B. Carter and P. Peter, "Dynamics and integrability property of the chiral string model," Physics Letters B, vol. 466, no. 1, pp. 41-49, 1999.

[141] T. Goto, "Relativistic quantum mechanics of one-dimensional mechanical continuum and subsidiary condition of dual resonance model," Progress of Theoretical Physics, vol. 46, no. 5, pp. 1560-1569, 1971.

[142] D. P. Bennett and F. R. Bouchet, "Cosmic-string evolution," Physical Review Letters, vol. 63, no. 26, pp. 2776-2779, 1989.

[143] A. Albrecht and N. Turok, "Evolution of cosmic string networks," Physical Review D, vol. 40, no. 4, pp. 973-1001, 1989.

[144] D. P. Bennett and F. R. Bouchet, "High-resolution simulations of cosmic-string evolution. I. Network evolution," Physical Review D, vol. 41, no. 8, pp. 2408-2433, 1990.

[145] B. Allen and E. P. S. Shellard, "Cosmic-string evolution: a numerical simulation," Physical Review Letters, vol. 64, no. 2, pp. 119-122, 1990.

[146] C. Ringeval, M. Sakellariadou, and F. R. Bouchet, "Cosmological evolution of cosmic string loops," Journal of Cosmology and Astroparticle Physics, vol. 2007, no. 2, article 23, 2007.

[147] C. J. A. P. Martins and E. P. S. Shellard, "Fractal properties and small-scale structure of cosmic string networks," Physical
Review D, vol. 73, no. 4, Article ID 043515, 6 pages, 2006.

[148] K. D. Olum and V. Vanchurin, "Cosmic string loops in the expanding universe," Physical Review D, vol. 75, no. 6, Article ID $063521,2007$.

[149] J. Urrestilla, N. Bevis, M. Hindmarsh, M. Kunz, and A. R. Liddle, "Cosmic microwave anisotropies from BPS semilocal strings," Journal of Cosmology and Astroparticle Physics, vol. 2008, no. 7, article 10, 2008.

[150] J. Urrestilla and A. Vilenkin, "Evolution of cosmic superstring networks: a numerical simulation," Journal of High Energy Physics, vol. 2008, no. 2, article 37, 2008.

[151] A. Hanany and K. Hashimoto, "Reconnection of colliding cosmic strings," Journal of High Energy Physics, vol. 2005, no. 6, article 21, 2005.

[152] A. Achúcarro and R. De Putter, "Effective nonintercommutation of local cosmic strings at high collision speeds," Physical Review D, vol. 74, no. 12, Article ID 121701, 2006.

[153] E. P. S. Shellard, "Cosmic string interactions," Nuclear Physics $B$, vol. 283, pp. 624-656, 1987.

[154] R. A. Matzner, "Interaction of $U(1)$ cosmic strings: numerical intercommutation," Computers in Physics, vol. 2, pp. 5164, 1988.

[155] L. M. A. Bettencourt and T. W. B. Kibble, "Non-intercommuting configurations in the collisions of type $I U(1)$ cosmic strings," Physics Letters B, vol. 332, no. 3-4, pp. 297-304, 1994.

[156] L. M. A. Bettencourt, P. Laguna, and R. A. Matzner, "Nonintercommuting cosmic strings," Physical Review Letters, vol. 78, no. 11, pp. 2066-2069, 1997.

[157] P. Salmi, A. Achúcarro, E. J. Copeland, T. W. B. Kibble, R. De Putter, and D. A. Steer, "Kinematic constraints on formation of bound states of cosmic strings: field theoretical approach," Physical Review D, vol. 77, no. 4, Article ID 041701, 2008.

[158] P. Laguna and R. A. Matzner, "Numerical simulation of bosonic-superconducting-string interactions," Physical Review D, vol. 41, no. 6, pp. 1751-1763, 1990.

[159] M. G. Jackson, N. T. Jones, and J. Polchinski, "Collisions of cosmic F- and D-strings," Journal of High Energy Physics, vol. 2005, no. 10, article 13, 2005.

[160] E. J. Copeland, T. W. B. Kibble, and D. A. Steer, "Collisions of strings with y junctions," Physical Review Letters, vol. 97, no. 2, Article ID 021602, 2006.

[161] E. J. Copeland, H. Firouzjahi, T. W. B. Kibble, and D. A. Steer, "Collision of cosmic superstrings," Physical Review D, vol. 77, Article ID 063521, 2008.

[162] N. Bevis and P. M. Saffin, "Cosmic string Y-junctions: a comparison between field theoretic and Nambu-Goto dynamics," Physical Review D, vol. 78, no. 2, Article ID 023503, 2008.

[163] P. Binetruy, A. Bohe, T. Hertog, and D .A. Steer, "Proliferation of sharp kinks on cosmic (super-)string loops with junctions," Journal of High Energy Physics, http://arxiv .org/abs/1005.2426.

[164] T. Vachaspati and A. Vilenkin, "Formation and evolution of cosmic strings," Physical Review D, vol. 30, no. 10, pp. 20362045, 1984.

[165] T. W. B. Kibble, “Configuration of $Z_{2}$ strings," Physics Letters $B$, vol. 166, no. 3, pp. 311-313, 1986.

[166] A. Yates and T. W. B. Kibble, "An extension to models for cosmic string formation," Physics Letters B, vol. 364, no. 3, pp. 149-156, 1995.

[167] J. Borrill, T. W. B. Kibble, T. Vachaspati, and A. Vilenkin, "Defect production in slow first-order phase transitions," Physical Review D, vol. 52, no. 4, pp. 1934-1943, 1995. 
[168] R. J. Scherrer and A. Vilenkin, "“Lattice-free” simulations of topological defect formation," Physical Review D, vol. 58, no. 10, Article ID 103501, 8 pages, 1998.

[169] R. J. Scherrer and A. Vilenkin, "Cosmic string formation from correlated fields," Physical Review D, vol. 56, no. 2, pp. 647-652, 1997.

[170] A. Vilenkin, "Gravitational radiation from cosmic strings," Physics Letters B, vol. 107, no. 1-2, pp. 47-50, 1981.

[171] T. Damour and A. Vilenkin, "Gravitational wave bursts from cusps and kinks on cosmic strings," Physical Review D, vol. 64, no. 6, Article ID 064008, 2001.

[172] X. Siemens, J. Creighton, I. Maor, S. R. Majumder, K. Cannon, and J. Read, "Gravitational wave bursts from cosmic (super)strings: quantitative analysis and constraints," Physical Review D, vol. 73, no. 10, Article ID 105001, 2006.

[173] S. Olmez, V. Mandic, and X. Siemens, "Gravitational-wave stochastic background from kinks and cusps on cosmic strings," Physical Review D, vol. 81, Article ID 104028, 10 pages, 2010.

[174] T. Vachaspati, "Cosmic sparks from superconducting strings," Physical Review Letters, vol. 101, no. 14, Article ID 141301, 2008.

[175] T. Vachaspati, "Cosmic rays from cosmic strings with condensates," Physical Review D, vol. 81, no. 4, Article ID 043531, 2010.

[176] D. Austin, E. J. Copeland, and T. W. B. Kibble, "Evolution of cosmic string configurations," Physical Review D, vol. 48, no. 12, pp. 5594-5627, 1993.

[177] E. J. Copeland, T. W. B. Kibble, and D. A. Steer, "Evolution of a network of cosmic string loops," Physical Review D, vol. 58, no. 4, Article ID 043508, 14 pages, 1998.

[178] C. J. A. P. Martins and E. P .S. Shellard, "Fractal properties and small-scale structure of cosmic string networks," Physical Review D, vol. 73, no. 4, Article ID 043515, 6 pages, 2006.

[179] J. Polchinski and J. V. Rocha, "Analytic study of small scale structure on cosmic strings," Physical Review D, vol. 74, no. 8, Article ID 083504, 2006.

[180] F. Dubath, J. Polchinski, and J. V. Rocha, "Cosmic string loops, large and small," Physical Review D, vol. 77, no. 12, Article ID 123528, 2008.

[181] E. J. Copeland and T. W. B. Kibble, "Kinks and small-scale structure on cosmic strings," Physical Review D, vol. 80, no. 12, Article ID 123523, 7 pages, 2009.

[182] J. V. Rocha, "Scaling solution for small cosmic string loops," Physical Review Letters, vol. 100, no. 7, Article ID 071601, 2008.

[183] B. Allen and E. P. S. Shellard, "Gravitational radiation from cosmic strings," Physical Review D, vol. 45, no. 6, pp. 18981912, 1992.

[184] X. Siemens, K. D. Olum, and A. Vilenkin, "Size of the smallest scales in cosmic string networks," Physical Review D, vol. 66, no. 4, Article ID 043501, 4 pages, 2002.

[185] G. R. Vincent, M. Hindmarsh, and M. Sakellariadou, "Scaling and small-scale structure in cosmic string networks," Physical Review D, vol. 56, no. 2, pp. 637-646, 1997.

[186] R. Durrer and Z.-H. Zhou, "Large-scale structure formation with global topological defects," Physical Review D, vol. 53, no. 10, pp. 5394-5410, 1996.

[187] N. Turok, "Causality and the Doppler peaks," Physical Review D, vol. 54, no. 6, pp. R3686-R3689, 1996.

[188] U.-L. Pen, U. Seljak, and N. Turok, "Power spectra in global defect theories of cosmic structure formation," Physical Review Letters, vol. 79, no. 9, pp. 1611-1614, 1997.
[189] R. Durrer and M. Kunz, "Cosmic microwave background anisotropies from scaling seeds: generic properties of the correlation functions," Physical Review D, vol. 57, no. 6, pp. R3199-R3203, 1998.

[190] C. Contaldi, M. Hindmarsh, and J. Magueijo, "Power spectra of the cosmic microwave background and density fluctuations seeded by local cosmic strings," Physical Review Letters, vol. 82, no. 4, pp. 679-682, 1999.

[191] J.-H. P. Wu, P. P. Avelino, E. P. S. Shellard, and B. Allen, "Cosmic strings, loops, and linear growth of matter perturbations," International Journal of Modern Physics D, vol. 11, no. 1, pp. 61-102, 2002.

[192] N. Bevis, M. Hindmarsh, M. Kunz, and J. Urrestilla, "CMB power spectrum contribution from cosmic strings using field-evolution simulations of the Abelian Higgs model," Physical Review D, vol. 75, no. 6, Article ID 065015, 2007.

[193] N. Bevis, M. Hindmarsh, M. Kunz, and J. Urrestilla, "CMB power spectra from cosmic strings: predictions for the Planck satellite and beyond," Journal of High Energy Physics, http://arxiv.org/abs/1005.2663.

[194] N. Bevis, M. Hindmarsh, M. Kunz, and J. Urrestilla, "CMB power spectrum contribution from cosmic strings using field-evolution simulations of the Abelian Higgs model," Physical Review D, vol. 75, no. 6, Article ID 065015, 22 pages, 2007.

[195] J. Magueijo, A. Albrecht, D. Coulson, and P. Ferreira, "Doppler peaks from active perturbations," Physical Review Letters, vol. 76, no. 15, pp. 2617-2620, 1996.

[196] N. Bevis, M. Hindmarsh, M. Kunz, and J. Urrestilla, "Fitting cosmic microwave background data with cosmic strings and inflation," Physical Review Letters, vol. 100, no. 2, Article ID 021301, 4 pages, 2008.

[197] D. P. Bennett, A. Stebbins, and F. R. Bouchet, "The implications of the COBE diffuse microwave radiation results for cosmic strings," Astrophysical Journal, vol. 399, no. 1, pp. L5L8, 1992.

[198] U.-L. Pen, D. N. Spergel, and N. Turok, "Cosmic structure formation and microwave anisotropies from global field ordering," Physical Review D, vol. 49, no. 2, pp. 692-729, 1994.

[199] B. Allen, R. R. Caldwell, S. Dodelson, L. Knox, E. P. S. Shellard, and A. Stebbins, "Cosmic microwave background anisotropy induced by cosmic strings on angular scales $\gtrsim$ 15'," Physical Review Letters, vol. 79, no. 14, pp. 2624-2627, 1997.

[200] M. Landriau and E. P. S. Shellard, "Fluctuations in the cosmic microwave background induced by cosmic strings: methods and formalism," Physical Review D, vol. 67, no. 10, Article ID 103512, 2003.

[201] M. Landriau and E. P.S. Shellard, "Large angle cosmic microwave background fluctuations from cosmic strings with a cosmological constant," Physical Review D, vol. 69, no. 2, Article ID 023003, 2004.

[202] A. A. Fraisse, C. Ringeval, D. N. Spergel, and F. R. Bouchet, "Small-angle CMB temperature anisotropies induced by cosmic strings," Physical Review D, vol. 78, no. 4, Article ID 043535, 2008.

[203] M. Hindmarsh, "Small-scale microwave background fluctuations from cosmic strings," Astrophysical Journal, vol. 431, no. 2, pp. 534-542, 1994.

[204] A. Stebbins and S. Veeraraghavan, "Beyond the small-angle approximation for MBR anisotropy from seeds," Physical Review D, vol. 51, no. 4, pp. 1465-1478, 1995. 
[205] J. R. Gott III, C. Park, R. Juszkiewicz et al., "Topology of microwave background fluctuations: theory," Astrophysical Journal, vol. 352, no. 1, pp. 1-14, 1990.

[206] K. Takahashi, A. Naruko, Y. Sendouda, D. Yamauchi, C.M. Yoo, and M. Sasaki, "Non-Gaussianity in the cosmic microwave background temperature fluctuations from cosmic (super-)strings," Journal of Cosmology and Astroparticle Physics, vol. 2009, no. 10, article 3, 2009.

[207] K. Falconer, Fractal Geometry, John Wiley \& Sons, Chichester, UK, 2006.

[208] M. P. Pompilio, F. R. Bouchet, G. Murante, and A. Provenzale, "Multifractal analysis of string-induced cosmic microwave background radiation anisotropies," Astrophysical Journal, vol. 449, no. 1, pp. 1-8, 1995.

[209] M. P. Hobson, A. W. Jones, and A. N. Lasenby, "Wavelet analysis and the detection of non-Gaussianity in the cosmic microwave background," Monthly Notices of the Royal Astronomical Society, vol. 309, no. 1, pp. 125-140, 1999.

[210] R. B. Barreiro and M. P. Hobson, "The discriminating power of wavelets to detect non-Gaussianity in the cosmic microwave background," Monthly Notices of the Royal Astronomical Society, vol. 327, no. 3, pp. 813-828, 2001.

[211] D. K. Hammond, Y. Wiaux, and P. Vandergheynst, "Wavelet domain Bayesian denoising of string signal in the cosmic microwave background," Monthly Notices of the Royal Astronomical Society, vol. 398, no. 3, pp. 1317-1332, 2009.

[212] M. Hindmarsh, C. Ringeval, and T. Suyama, "CMB temperature bispectrum induced by cosmic strings," Physical Review $D$, vol. 80, no. 8, Article ID 083501, 2009.

[213] D. N. Spergel and D. M. Goldberg, "Microwave background bispectrum. I. Basic formalism," Physical Review D, vol. 59, no. 10, Article ID 103001, 8 pages, 1999.

[214] N. Aghanim, M. Kunz, P. G. Castro, and O. Forni, "Nongaussianity: comparing wavelet and Fourier based methods," Astronomy and Astrophysics, vol. 406, no. 3, pp. 797-816, 2003.

[215] M. Hindmarsh, C. Ringeval, and T. Suyama, "CMB temperature trispectrum of cosmic strings," Physical Review D, vol. 81, no. 6, Article ID 063505, 2010.

[216] N. Bartolo, E. Komatsu, S. Matarrese, and A. Riotto, "NonGaussianity from inflation: theory and observations," Physics Reports, vol. 402, no. 3-4, pp. 103-266, 2004.

[217] K. M. Smith, L. Senatore, and M. Zaldarriaga, "Optimal limits on $f_{N L}^{\text {local }}$ from WMAP 5-year data," Journal of Cosmology and Astroparticle Physics, vol. 2009, no. 9, 2009.

[218] D. M. Regan and E. P. S. Shellard, "General CMB and primordial trispectrum estimatio," Journal of Cosmology and Astroparticle Physics, http://arxiv.org/abs/1004.2915.

[219] M. Sakellariadou, "A note on the evolution of cosmic string/superstring networks," Journal of Cosmology and Astroparticle Physics, no. 4, article 3, pp. 103-116, 2005.

[220] A. Avgoustidis and E. P. S. Shellard, "Velocity-dependent models for non-Abelian/entangled string networks," Physical Review D, vol. 78, no. 10, Article ID 103510, 21 pages, 2008.

[221] D. M. Regan and E. P. S. Shellard, "Cosmic string power spectrum, bispectrum and trispectrum," Journal of Cosmology and Astroparticle Physics, http://arxiv.org/abs/0911.2491.

[222] M. Landriau and E. P. S. Shellard, "Cosmic string induced CMB maps," Journal of Cosmology and Astroparticle Physics, http://arxiv.org/abs/1004.2885. 

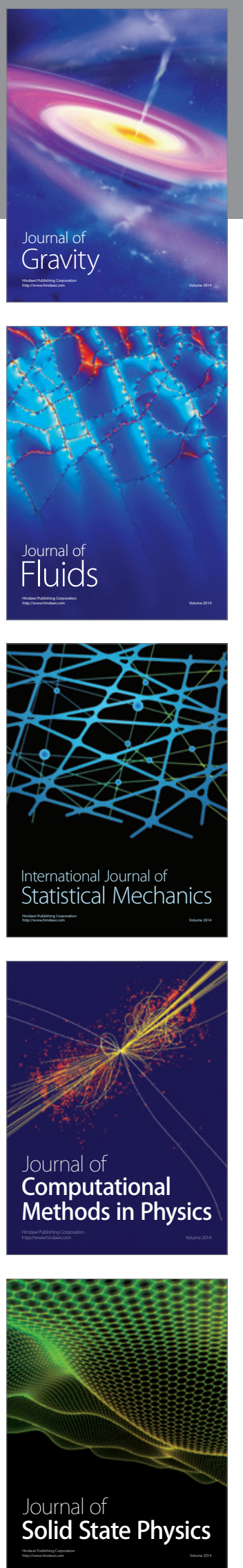

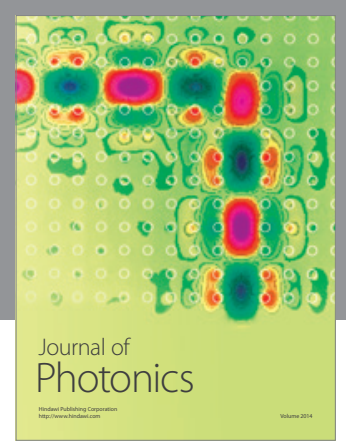

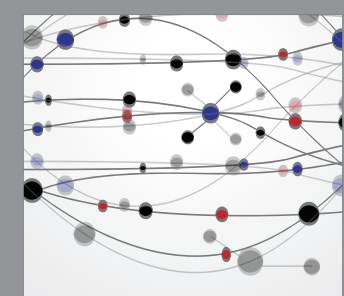

The Scientific World Journal
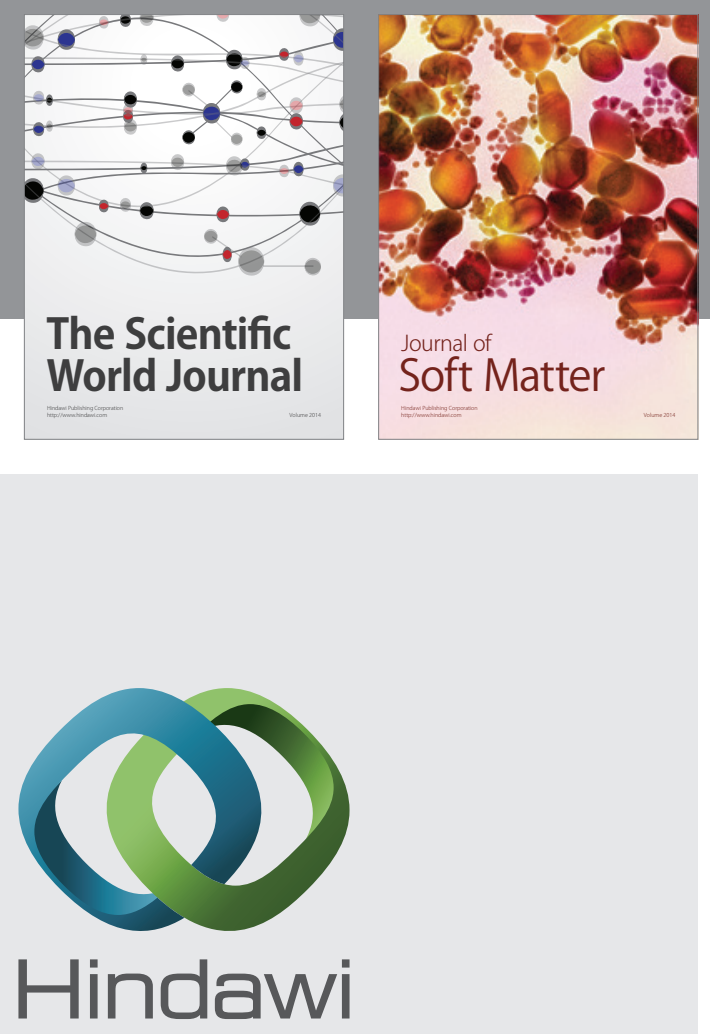

Submit your manuscripts at

http://www.hindawi.com
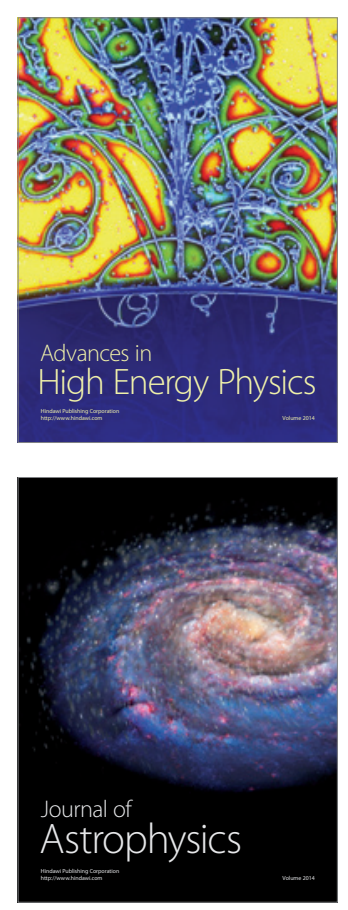
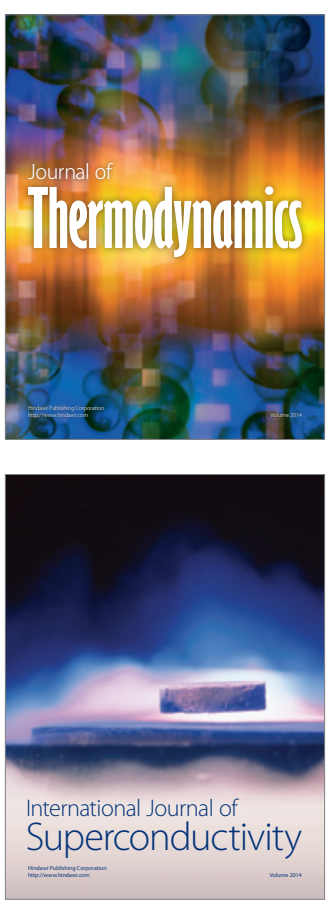
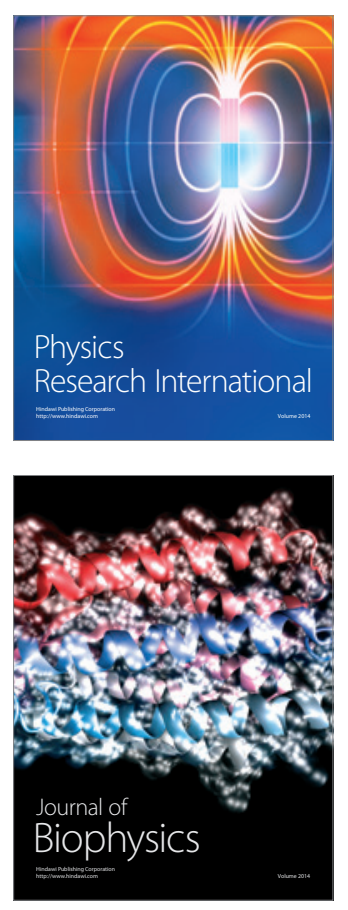
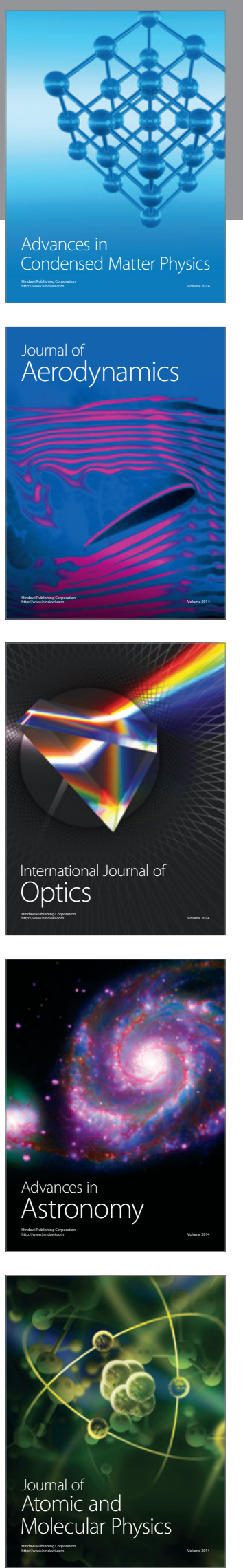